\title{
Genome-wide analysis of chicken snoRNAs provides unique implications for the evolution of vertebrate snoRNAs Peng Shao ${ }^{\dagger}$, Jian-Hua Yang ${ }^{\dagger}$, Hui Zhou, Dao-Gang Guan and Liang-Hu Qu*
}

Address: Key Laboratory of Gene Engineering of the Ministry of Education, State Key Laboratory for Biocontrol, Zhongshan University, Guangzhou 510275, PR China

Email: Peng Shao - lsssp@mail.sysu.edu.cn; Jian-Hua Yang - yjhua2110@yahoo.com.cn; Hui Zhou - lsszh@mail.sysu.edu.cn; DaoGang Guan - guanyufeirzz@sohu.com; Liang-Hu Qu* - lssqlh@mail.sysu.edu.cn

* Corresponding author †Equal contributors

Published: 22 February 2009

BMC Genomics 2009, 10:86 doi:10.1186/147|-2164-10-86
Received: I August 2008

Accepted: 22 February 2009

This article is available from: http://www.biomedcentral.com/I47/-2/64//0/86

(C) 2009 Shao et al; licensee BioMed Central Ltd.

This is an Open Access article distributed under the terms of the Creative Commons Attribution License (http://creativecommons.org/licenses/by/2.0), which permits unrestricted use, distribution, and reproduction in any medium, provided the original work is properly cited.

\begin{abstract}
Background: Small nucleolar RNAs (snoRNAs) represent one of the largest groups of functionally diverse trans-acting non-protein-coding (npc) RNAs currently known in eukaryotic cells. Chicken snoRNAs have been very poorly characterized when compared to other vertebrate snoRNAs. A genome-wide analysis of chicken snoRNAs is therefore of great importance to further understand the functional evolution of snoRNAs in vertebrates.

Results: Two hundred and one gene variants encoding 93 box $C / D$ and 62 box H/ACA snoRNAs were identified in the chicken genome and are predicted to guide 862 '-O-ribose methylations and 69 pseudouridylations of rRNAs and spliceosomal RNAs. Forty-four snoRNA clusters were grouped into four categories based on synteny characteristics of the clustered snoRNAs between chicken and human. Comparative analyses of chicken snoRNAs revealed extensive recombination and separation of guiding function, with cooperative evolution between the guiding duplexes and modification sites. The gas5-like snoRNA host gene appears to be a hotspot of snoRNA gene expansion in vertebrates. Our results suggest that the chicken is a good model for the prediction of functional snoRNAs, and that intragenic duplication and divergence might be the major driving forces responsible for expansion of novel snoRNA genes in the chicken genome.
\end{abstract}

Conclusion: We have provided a detailed catalog of chicken snoRNAs that aids in understanding snoRNA gene repertoire differences between avians and other vertebrates. Our genome-wide analysis of chicken snoRNAs improves annotation of the 'darkness matter' in the npcRNA world and provides a unique perspective into snoRNA evolution in vertebrates.

\section{Background}

The term small nucleolar RNAs (snoRNAs) was originally coined to describe the nucleolar localization of this group of RNAs relative to the other small nucleoplasmic RNAs. In sharp contrast to the relatively low abundance spliceosomal nuclear RNA (snRNA) species, snoRNAs represent one of the largest groups of functionally diverse trans-act- ing non-protein-coding RNAs (npcRNAs) currently known in eukaryotic cells [1,2]. On the basis of conserved sequence elements and characteristic secondary structures, snoRNAs can be divided into two major classes, box $\mathrm{C} / \mathrm{D}$ and box H/ACA snoRNAs. Box C/D snoRNAs contain two conserved motifs, the $5^{\prime}$ end box C (RUGAUGA, where $\mathrm{R}$ stands for any purine) and the $3^{\prime}$ end box $\mathrm{D}$ 
(CUGA). Box H/ACA snoRNAs exhibit a common hairpin-hinge-hairpin-tail secondary structure with the $\mathrm{H}$ box (ANANNA, where $\mathrm{N}$ stands for any nucleotide) in the hinge region and the ACA motif three nucleotides from the 3 ' end of the molecule. During the post-transcriptional processing of diverse RNAs most members of the known C/D and H/ACA snoRNAs respectively guide 2'-Oribose methylation and pseudouridylation $(\Psi)$. Recently, a new class of guide RNAs has been found to accumulate in the small Cajal body [3] and are thus termed small Cajal body-specific RNAs (scaRNAs). scaRNAs are often composed of both C/D box and H/ACA box domains [4] and guide the modification of RNA-polymerase-II-transcribed snRNAs [3]. Remarkably, an increasing number of 'orphan' snoRNAs lacking antisense to known RNA targets have been identified [5]. Many of them exhibit a tissuespecific or restricted expression pattern $[6,7]$ and are linked to genomic imprinting [6].

Interestingly, various snoRNA gene organizations have been characterized in different organisms [5,8]. Most snoRNAs are encoded in the introns of protein-coding or non-protein-coding genes in vertebrates [9]. Many snoRNA paralogs are usually clustered in different introns of the same host genes (HGs) or in the introns of different HGs by intragenic or intergenic duplication (including retroposition) from existing snoRNAs $[7,10-13]$, respectively. The distinct character of clustering gene organizations and evolutionary conservation of vertebrate snoRNAs facilitates detection of snoRNA homologs by sequence similarity alone in the genome [14]. However, many other snoRNAs in mammals cannot be found by simple homology search.

To date, hundreds of snoRNAs have been identified in mammals $[7,13,15-18]$ by approaches including computational and experimental RNomics. Although a limited number of snoRNAs were predicted in the chicken (Gallus gallus) genome by similarity search [19], the nature of chicken snoRNAs is poorly understood when compared with other vertebrates and their numbers far underrepresented. Additionally, detailed information on snoRNA guiding functions, genomic organization and evolution in the chicken genome is still unavailable. As a typical amniote, the chicken has evolved separately from mammals for about 310 million years [19]. The identification of chicken snoRNAs using conventional prediction methods such as a similarity search might be hindered by the sufficient nucleotide variation occurring in the genome. Recently, we developed an advanced computational package snoSeeker for the specific detection of guide box C/D (CDseeker) and box H/ACA (ACAseeker) snoRNAs, as well as orphan snoRNA genes in the human genome [7]. In the present work, 93 box $\mathrm{C} / \mathrm{D}$ and 62 box H/ACA snoRNAs have been identified in the chicken genome by apply- ing the computational package and experimental methods based on RT-PCR. The characteristics of the guiding function and genomic organization of the chicken snoRNAs have been extensively compared with the human counterparts. As a result, we provide for the first time a detailed catalog of chicken snoRNAs that facilitates understanding of snoRNA gene repertoire differences between the avian and other vertebrate lineages.

\section{Results \\ Computational identification of box CID snoRNA genes from G. gallus}

The CDseeker program was applied to search the G.gallus genome for box C/D snoRNAs. In total, 132 gene variants encoding 83 box C/D snoRNAs with the ability to guide 2 '-O-ribose methylation at 86 residues in rRNAs and snRNAs and 10 orphan box C/D snoRNAs were identified from the G.gallus genome (Table 1, see Additional file 1 and 2). Sixty-five box C/D snoRNAs are singleton. The other 28 snoRNAs have undergone one or more duplications in the chicken, which account for 67 paralogs. The majority of these RNAs (73 snoRNAs) have been assigned to guide only one methylation of the rRNAs or snRNAs, and are known as single-guide snoRNAs. Seventy-one snoRNAs uniquely guide methylations of the rRNAs, and 12 box C/D RNAs are predicted to guide methylation in the snRNAs or both the rRNAs and snRNAs. Interestingly, two methylation sites at $18 \mathrm{~S}$ rRNA-C757 and U2-U47 (corresponding to human 18S rRNA-C797 and U2-U47), which have been previously reported to lack potential guide snoRNAs, were predicted to be guided by GGgCD20 and GGgCD76, respectively. Among the 83 guide RNAs, only a fraction of box C/D snoRNAs ( $15 \%)$ are doubleguide snoRNAs. A comparative analysis of these chicken box C/D snoRNAs and their counterparts at the corresponding genomic loci in six other vertebrate genomes (human, mouse, opossum, platypus, lizard and frog) revealed that 13 snoRNA genes appeared to be specific to the chicken or avian lineage, and the remainder had their cognate snoRNAs in at least one other vertebrate genome (see Additional file 3). In total, 55 chicken box C/D snoRNA genes are conserved in amniotic species. These snoRNAs are assigned as the core amniotic box C/D snoRNAs, out of which 31 box C/D snoRNA genes are the core vertebrate box $C / D$ snoRNAs conserved in vertebrates (Figure 1A).

\section{Computational identification of box HIACA snoRNA genes from G. gallus}

Based on the conserved 'hairpin-hinge-hairpin-tail' structure and the $\mathrm{H}$ and ACA/ATA box motifs, the ACAseeker program was performed to identify chicken box H/ACA snoRNA genes. In total, 69 RNA variants encoding 52 guide and 10 orphan box H/ACA snoRNAs were identified in the chicken genome (Table 2, see Additional file 2). 


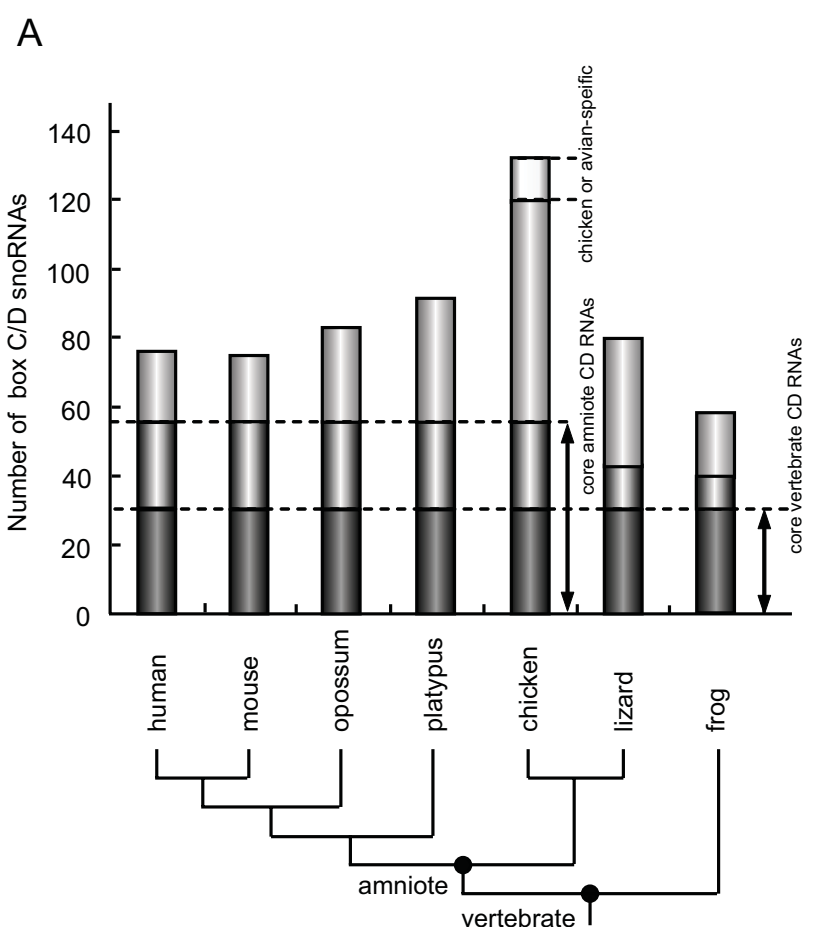

$\square$ core vertebrate snoRNAs
$\square$ pairwise orthologs between chicken and the other speices
B

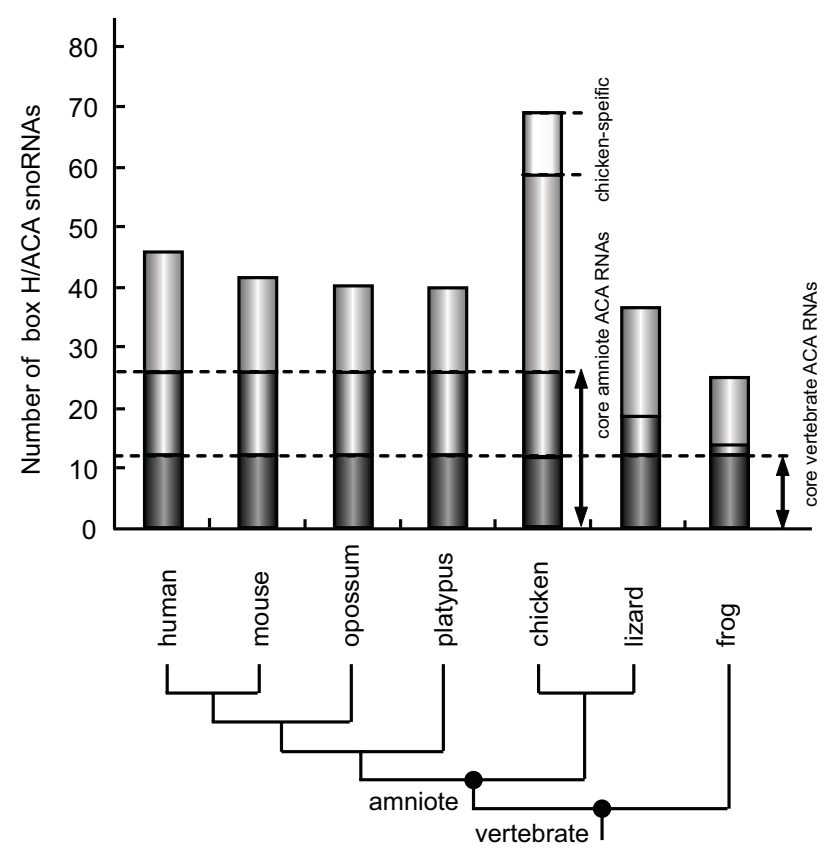

core amniote snoRNAs
$\square$ chicken or avian-specific snoRNAs

Figure I

Chicken snoRNAs classified according to their predicted evolutionary relationships with cognate snoRNAs of six other model vertebrates (human, mouse, opossum, platypus, lizard and frog). (A) box C/D snoRNAs. (B) box H/ACA snoRNAs. Pairwise orthologs are assigned when orthology is detectable in the chicken and one other vertebrate genome.

Compared with the chicken box C/D snoRNAs, a higher percentage of box H/ACA snoRNAs ( $90 \%)$ are singleton. Only six snoRNAs had undergone one or more duplications, yielding 13 paralogs. Fifty-two guide box H/ACA RNAs were predicted to guide 69 \%s in rRNAs and snRNAs (see Additional file 1). The majority of these RNAs (42 snoRNAs) have been assigned to guide $\Psi$ s of the rRNAs. Nine scaRNAs have been predicted to guide $\Psi$ s of the snRNAs (GGgACA48 52) or both the rRNAs and snRNAs (GGgACA10, GGgACA14, GGgACA18 and GGgACA29).

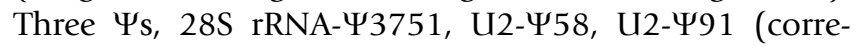

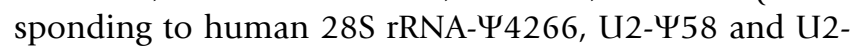

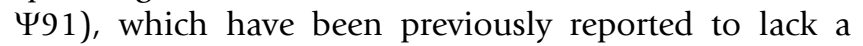
potential guide snoRNA, were predicted to be guided by GGgACA43, GGgACA18 and GGgACA10, respectively. In sharp contrast to the box C/D snoRNAs, approximately half of the H/ACA snoRNAs are capable of directing more than one $\Psi$ which are often located on the same rRNA or snRNA. Interestingly, GGgACA5 and GGgACA6 show the potential of directing three nonadjacent $\Psi$ s by a single guide sequence and are the first to be reported in the chicken. In the case of GGgACA51, whose sequence shows high similarity to that of half of the U93 composed of two tandem arranged box H/ACA RNA domains [20], it should be annotated as the half U93 homolog present in humans. Comparison of the conserved regions encoding snoRNAs in the chicken and six other vertebrate genomes revealed that eight are found to be chicken-specific, and 26 are the core amniotic box H/ACA snoRNA genes which include 12 core vertebrate snoRNA genes (Figure 1B, see Additional file 3).

\section{Experimental confirmation of the computational results}

Recently, PCR-based methods have been successfully used for small RNA detection, as well as expression profiling $[21,22]$. In this study, we developed an improved method for specifically detecting the expression of snoRNA candidates (see Additional file 4). As different expression levels might be detected for snoRNAs in different host genes, we first detected the expression of 14 guide snoRNAs located in different host genes to test the method we developed (Figure 2A). In most cases, unique and obvious bands 
Table I: Box C/D snoRNAs in chicken

\begin{tabular}{|c|c|c|c|c|c|c|c|c|}
\hline \multirow{2}{*}{$\begin{array}{l}\text { SnoRNA } \\
\text { ID' }\end{array}$} & \multirow[t]{2}{*}{ Iso } & \multirow{2}{*}{$\begin{array}{l}\text { Len } \\
\text { (nt) }\end{array}$} & \multirow[t]{2}{*}{ Modification } & \multirow[t]{2}{*}{ Antisense element } & \multicolumn{3}{|c|}{ Functional homologs } & \multirow[t]{2}{*}{ Host gene/annotation ${ }^{2}$} \\
\hline & & & & & Yeast & Plant & Mammal & \\
\hline GGgCDI & I & 98 & $5.8 \mathrm{~S}-\mathrm{U}-14$ & $13 \mathrm{nt}\left(5^{\prime}\right)$ & & & HBII-239 & APIGI (intron II) \\
\hline GGgCD2 & I & 91 & 18S-A-99 & 13 nt (3') & snR5I & & U57 & NOL5A (intron 9) \\
\hline \multirow[t]{2}{*}{ GGgCD3 } & I & 83 & $18 S-U-116$ & 12 nt (3') & & & $\mathrm{U} 42 \mathrm{~A} / \mathrm{B}$ & RPL23A (intron 3) \\
\hline & & & U4-C-8 & II nt (5') & & & U9I/mgUI2-22/U4-8 & \\
\hline $\mathrm{GGgCD} 4$ & I & 84 & $|8 S-U-| 2 \mid$ & I I nt (5') & & & $\mathrm{ZI7a} / \mathrm{b} / \mathrm{mgh} \mid 8 \mathrm{~s}-121$ & RPL23A (intron 2) \\
\hline \multirow{2}{*}{ GGgCD5 } & 2 & 103 & $18 S-A-158$ & 17 nt (3') & & & $\mathrm{U} 45 \mathrm{~A} / \mathrm{C}$ & RABGGTB (intron I,2) \\
\hline & & & $|8 S-U-| 7 \mid$ & 13 nt (5') & & & U $45 \mathrm{~A} / \mathrm{C}$ & \\
\hline GGgCD6 & I & 90 & $18 S-A-165$ & 10 nt (3') & & & U44 & mRNA CR387333 (intron 7) \\
\hline GGgCD7 & i & 96 & I8S-U-389 & $12 \mathrm{nt}\left(5^{\prime}\right)$ & & & HBII-202 & RPLI3 (intron 2) \\
\hline GGgCD8 & i & 100 & I8S-G-397 & $10 \mathrm{nt}\left(5^{\prime}\right)$ & & & HBII-429 & RPSI2 (intron 4) \\
\hline \multirow[t]{2}{*}{ GGgCD9 } & 3 & 113 & I8S-C-423 & 14 nt (3') & UI4 & UI4 & UI4A/B & HSPA8 (intron $5,6,8$ ) \\
\hline & & & $28 \mathrm{~S}-\mathrm{C}-3358$ & II nt (3') & & snoR37 & U53/snoRDI22 & \\
\hline \multirow[t]{2}{*}{ GGgCDI0 } & 2 & 109 & $18 \mathrm{~S}-\mathrm{A}-429$ & $13 \mathrm{nt}\left(3^{\prime}\right)$ & snR52 & snoR52Y & U83a/b & $\begin{array}{l}\text { LOC77I } 432 \text { (intron I); } \\
\text { chrl_II.94 (intron 8) }\end{array}$ \\
\hline & & & $28 \mathrm{~S}-\mathrm{C}-3190$ & II nt (5') & snR76 & & HBII-I80a/b/c & \\
\hline GGgCDII & 2 & 124 & I8S-A-445 & II nt (5') & snR87 & snoRI5 & UI6 & RPL4 (intron I,3) \\
\hline GGgCDI 2 & 2 & 99 & $18 S-G-470$ & $12 \mathrm{nt}\left(3^{\prime}\right)$ & & & HBII-95/snoRDIIB & NOP5/58 (intron 8,9) \\
\hline GGgCDI3 & 2 & 95 & I8S-A-473 & 12 nt (3') & & & HBII-234 & NOP5/58 (intron 3,6) \\
\hline GGgCDI4 & I & 91 & $|8 S-A-55|$ & $12 \mathrm{nt}\left(5^{\prime}\right)$ & snR4I & snoR4IY & U62A/B & LOC4I7I77 (intron 8) \\
\hline GGgCDI5 & 3 & 104 & 18S-G-562 & II nt (5') & & & HBII-25I/UI03 & PUMI (intron 9,12,21) \\
\hline GGgCDI6 & I & 101 & I8S-U-588 & 13 nt (5') & snR77 & & HBII-I 35 & mRNA CR391615 (intron 4) \\
\hline GGgCDI7 & i & 86 & I8S-G-605 & I I nt (3') & & U54 & U54 & RPS20 (intron 3) \\
\hline \multirow[t]{2}{*}{ GGgCDI8 } & 2 & 98 & $18 \mathrm{~S}-\mathrm{A}-629$ & 14 nt (3') & snR47 & U36la & U36a/B & RPL7A (intron 3,4) \\
\hline & & & $28 \mathrm{~S}-\mathrm{A}-3213$ & 13 nt (5') & snR47 & U36lla & U36C & \\
\hline $\mathrm{GGgCD} 19$ & 3 & 89 & I8S-G-644 & I I nt (3') & & & HBII-I08 & GNL3 (intron 5,7,9) \\
\hline GGgCD20 & I & 84 & 18S-C-757 & II nt (3') & & & & COX7C (intron I) \\
\hline $\mathrm{GGgCD} 21$ & 2 & 86 & 18S-G-825 & II nt (5') & & & HBII-4I9 & $\begin{array}{l}\text { CCARI (intron II); } \\
\text { AP2BI (intron I6) }\end{array}$ \\
\hline GGgCD22 & I & 93 & 18S-A-989 & 17 nt (5') & snR54 & U59 & U59A/B & ATP5B (intron 2) \\
\hline $\mathrm{GGgCD} 23$ & 2 & 99 & $18 \mathrm{~S}-\mathrm{C}-1230$ & $12 \mathrm{nt}\left(5^{\prime}\right)$ & & & HBII-I42 & EIF4G I (intron II, I4) \\
\hline GGgCD24 & i & 92 & I8S-U-I 246 & $13 \mathrm{nt}\left(5^{\prime}\right)$ & & & HBII-55 & NOL5A (intron 6) \\
\hline GGgCD25 & 2 & 89 & |8S-G-1286 & $12 \mathrm{nt}\left(5^{\prime}\right)$ & snR40 & snR2I & U32A/B & KPNBI (intron 4,7) \\
\hline $\mathrm{GGgCD} 26$ & I & 89 & 18S-U-1397 & $12 \mathrm{nt}\left(3^{\prime}\right)$ & & U6I & U6I & RBMX (intron 2) \\
\hline GGgCD27 & 1 & 101 & |8S-G-1402 & $10 \mathrm{nt}\left(3^{\prime}\right)$ & & & SNORDII8 & PRPF39 (intron 10) \\
\hline GGgCD28 & I & 88 & |8S-A-1633 & II nt (5') & & & U82/Z25 & NCL (intron 5) \\
\hline GGgCD29 & 2 & 101 & 18S-C-1659 & $10 \mathrm{nt}\left(5^{\prime}\right)$ & snR70 & U43 & U43 & chrl_Il.94 (intron 3,6) \\
\hline GGgCD30 & I & 98 & I8S-U-1758 & $18 \mathrm{nt}\left(3^{\prime}\right)$ & & & $\mathrm{U} 20$ & $\mathrm{NCL}$ (intron II) \\
\hline $\mathrm{GGgCD} 31$ & i & 93 & $28 S-A-413$ & $13 \mathrm{nt}\left(5^{\prime}\right)$ & & & U8I/Z23 & $\begin{array}{l}\text { mRNA CR387333 } \\
\text { (intron I2) }\end{array}$ \\
\hline GGgCD32 & I & 115 & $28 S-G-1253$ & 13 nt (3') & & & U2I & RPL5 (intron 4) \\
\hline GGgCD33 & 3 & 94 & $28 \mathrm{~S}-\mathrm{A}-1263$ & 13 nt (5') & UI8 & UI8 & $\mathrm{U} / 8 \mathrm{a} / \mathrm{b} / \mathrm{c}$ & RPL4 (intron 2,5,6) \\
\hline GGgCD34 & 1 & 88 & $28 \mathrm{~S}-\mathrm{G}-1457$ & $19 \mathrm{nt}\left(5^{\prime}\right)$ & snR39b & snoR39BY & snR39B & EIF4A2 (intron 3) \\
\hline GGgCD35 & i & 95 & $28 \mathrm{~S}-\mathrm{A}-1459$ & 14 nt (5') & snR39/snR59 & U5I & U32A/B/U5I & EEFIB2 (intron 3) \\
\hline \multirow[t]{2}{*}{ GGgCD36 } & 2 & 98 & $28 \mathrm{~S}-\mathrm{A}-1469$ & $9 \mathrm{nt}\left(5^{\prime}\right)$ & $\operatorname{snR} 60$ & U80 & U77/80 & $\begin{array}{l}\text { mRNA CR387333 } \\
\text { (intron 4,5) }\end{array}$ \\
\hline & & & $28 S-G-1560$ & 15 nt (3') & snR60 & U80 & U80/ZI5 & \\
\hline GGgCD37 & 2 & 92 & $28 \mathrm{~S}-\mathrm{A}-1792$ & I I nt (5') & snR6I & U38 & U38a/b & RPS8 (intron 4,5) \\
\hline \multirow[t]{2}{*}{ GGgCD38 } & I & 92 & $28 S-C-2112$ & 12 nt (3') & U24 & U24 & U24 & RPL7A (intron I) \\
\hline & & & $28 \mathrm{~S}-\mathrm{C}-2126$ & I I nt (5') & & & U24 & \\
\hline GGgCD39 & I & 104 & $28 \mathrm{~S}-\mathrm{A}-2124$ & $9 \mathrm{nt}\left(5^{\prime}\right)$ & U24 & U24 & U76/Z20 & mRNA CR387333 (intron 3) \\
\hline GGgCD40 & 1 & 92 & $28 \mathrm{~S}-\mathrm{C}-2183$ & $13 \mathrm{nt}\left(5^{\prime}\right)$ & & & mgh28S-2409 & $\begin{array}{l}\text { ENSGALESTG00000033205 } \\
\text { (intron 5) }\end{array}$ \\
\hline GGgCD4I & I & 91 & $28 \mathrm{~S}-\mathrm{G}-2 \mid 85$ & 13 nt (5') & & & mgh28S-24II & $\begin{array}{l}\text { ENSGALESTG00000033205 } \\
\text { (intron 8) }\end{array}$ \\
\hline GGgCD42 & 1 & 86 & $28 \mathrm{~S}-\mathrm{A}-2548$ & 13 nt (3') & & & HBII-420 & EST CK9868I0 (intron 4) \\
\hline GGgCD43 & i & 100 & $28 \mathrm{~S}-\mathrm{C}-2565$ & 10 nt (3') & & snoRI5 & U39/U55 & RPS8 (intron I) \\
\hline GGgCD44 & I & 93 & 28S-A-2576 & 13 nt (3') & & & U95 & GNB2LI (intron 6) \\
\hline GGgCD45 & i & 102 & $28 \mathrm{~S}-\mathrm{A}-3207$ & 17 nt (5') & & & U37 & EEF2 (intron 6) \\
\hline GGgCD46 & 3 & 92 & $28 \mathrm{~S}-\mathrm{G}-3233$ & I I nt (5') & & & HBII-276 & EST BU30826I (intron I,2,3) \\
\hline GGgCD47 & 2 & 122 & $28 \mathrm{~S}-\mathrm{A}-3249$ & II nt (3') & snR63 & U40 & U40/U46 & RPS8 (intron 2,3) \\
\hline
\end{tabular}


Table I: Box C/D snoRNAs in chicken (Continued)

\begin{tabular}{|c|c|c|c|c|c|c|c|c|}
\hline GGgCD48 & 3 & 158 & $28 \mathrm{~S}-\mathrm{A}-3274$ & $10 \mathrm{nt}\left(3^{\prime}\right)$ & snRI3 & U15 & UI5a/b & RPS3 (intron 4,5,6) \\
\hline GGgCD49 & I & 90 & 28S-A-3274 & $10 \mathrm{nt}\left(5^{\prime}\right)$ & $\operatorname{snR} 13$ & UI5 & UI5a/b & RFWD2 (intron 6) ${ }^{3}$ \\
\hline GGgCD50 & 2 & 269 & 28S-U-3307 & $12 \mathrm{nt}\left(5^{\prime}\right)$ & & & $\mathrm{HBI}-43$ & DKCI (intron 4,8) \\
\hline GGgCD5I & I & 95 & $28 \mathrm{~S}-\mathrm{A}-3319$ & I3 nt (5') & & snoR44 & U79/Z22 & mRNA CR387333 (intron 9) \\
\hline GGgCD52 & I & 90 & $28 \mathrm{~S}-\mathrm{C}-3330$ & 12 nt (5') & snR64 & snoR44 & U74/ZI8 & mRNA CR387333 (intron I) \\
\hline GGgCD53 & 2 & 106 & 28S-A-3356 & 14 nt (3') & & & HBII-3I6 & WDR43 (intron 4,5) \\
\hline GGgCD54 & 2 & 101 & 28S-C-3358 & $13 \mathrm{nt}\left(3^{\prime}\right)$ & & snoR37 & U53/snoRDI22 & WDR43(intron 3,9) \\
\hline GGgCD55 & 1 & 117 & $28 \mathrm{~S}-\mathrm{C}-3358$ & $10 \mathrm{nt}\left(5^{\prime}\right)$ & & & U53/snoRDI 22 & NOL5A (intron 7) \\
\hline GGgCD56 & $\mathrm{I}$ & 97 & $28 S-C-3376$ & $12 \mathrm{nt}\left(5^{\prime}\right)$ & & & U47 & $\begin{array}{l}\text { mRNA CR387333 } \\
\text { (intron II) }\end{array}$ \\
\hline GGgCD57 & 4 & 104 & 28S-G-3388 & $12 \mathrm{nt}\left(5^{\prime}\right)$ & $\operatorname{snR} 190$ & & HBII-99 & $\begin{array}{l}\text { EST BU28I079 } \\
\text { (intron I,2,3,4) }\end{array}$ \\
\hline GGgCD58 & 2 & 94 & 28S-G-3433 & 14 nt (5') & & & HBII-82/snoRDIIIB & SF3B3 (intron 3,6) \\
\hline GGgCD59 & I & 96 & 28S-G-3502 & II nt (3') & & & UI02 & RPL2I (intron 3) \\
\hline GGgCD60 & I & 89 & $28 \mathrm{~S}-\mathrm{C}-3514$ & $13 \mathrm{nt}\left(5^{\prime}\right)$ & & & U75 & mRNA CR387333 (intron 2) \\
\hline \multirow[t]{2}{*}{ GGgCD6I } & 5 & 89 & $28 S-U-3682$ & $12 \mathrm{nt}\left(5^{\prime}\right)$ & Z20 & snoRI0 & U58a/b & $\begin{array}{l}\text { RPLI7 (intron 4,5,6); mRNA } \\
\text { CR352619 (intron 4, 5) }\end{array}$ \\
\hline & & & $28 S-G-3683$ & $12 \mathrm{nt}\left(5^{\prime}\right)$ & & & U58a/b & \\
\hline GGgCD62 & I & 89 & $28 S-G-3683$ & $12 \mathrm{nt}\left(5^{\prime}\right)$ & & & U58a/b & RPLI7 (intron I) \\
\hline GGgCD63 & I & 108 & 28S-G-3825 & $14 \mathrm{nt}\left(5^{\prime}\right)$ & snR48 & & U60 & Chrl4_2.70 (intron 9) ${ }^{3}$ \\
\hline GGgCD64 & I & 92 & 28S-G-3847 & II nt (5') & $\operatorname{snR} 38$ & snoR38Y & $\operatorname{snR} 38 \mathrm{a} / \mathrm{b} / \mathrm{c}$ & $\begin{array}{l}\text { ENSGALG0000000I886 } \\
\text { (intron 2) }\end{array}$ \\
\hline GGgCD65 & I & 100 & $28 \mathrm{~S}-\mathrm{C}-3911$ & $12 \mathrm{nt}\left(5^{\prime}\right)$ & & & $\mathrm{U} 49 \mathrm{a} / \mathrm{b}$ & mRNA CR391615 (intron 2) \\
\hline GGgCD66 & I & 87 & 28S-G-3949 & $10 \mathrm{nt}\left(3^{\prime}\right)$ & & & HBII-2IO & GNL3 (intron 8) \\
\hline GGgCD67 & I & 109 & 28S-C-3991 & $13 \mathrm{nt}\left(5^{\prime}\right)$ & snR73 & U35 & U35A/B & RPSI I (intron 4) \\
\hline GGgCD68 & I & 91 & $28 \mathrm{~S}-\mathrm{A}-4026$ & $12 \mathrm{nt}\left(5^{\prime}\right)$ & & & U63 & HSPA9 (intron II) \\
\hline GGgCD69 & 2 & 100 & $28 S-G-4073$ & 14 nt (5') & & & HBII-296a/b/c & TSRI (intron 8,II) \\
\hline \multirow[t]{2}{*}{ GGgCD70 } & I & 94 & 28S-U-4075 & I3 nt (5') & & & HBII-240 & RPL37 (intron 3) \\
\hline & & & 28S-G-4078 & II nt (5') & & & U78 & \\
\hline GGgCD7I & I & 89 & 28S-G-4078 & I I nt (5') & & & U78 & mRNA CR387333 (intron 8) \\
\hline GGgCD72 & 2 & 92 & 28S-G-4092 & $12 \mathrm{nt}\left(5^{\prime}\right)$ & & & SNORDI2I & UBAP2 (intron 13,15 ) \\
\hline GGgCD73 & I & 84 & UI-A-70 & $21 \mathrm{nt}\left(3^{\prime}\right)$ & & & U90 & chrl2_I.25 (intron 10) ${ }^{3}$ \\
\hline GGgCD74 & 1 & 130 & U2-G-12 & $10 \mathrm{nt}\left(5^{\prime}\right)$ & & & HBII-289 & mRNA CR389573 (intron I) ${ }^{3}$ \\
\hline GGgCD75 & I & 87 & U2-A-30 & $10 \mathrm{nt}\left(3^{\prime}\right)$ & & & Z32/mgU2-19_30 & chrI_38.I56 (intron 23) \\
\hline GGgCD76 & I & 160 & U2-U-47 & $13 \mathrm{nt}\left(3^{\prime}\right)$ & & & & TRRAP (intron I) \\
\hline GGgCD77 & I & 109 & U4-C-8 & $13 \mathrm{nt}\left(3^{\prime}\right)$ & & & U91/mgU/2-22/U4-8 & RFWD2 (intron II) \\
\hline \multirow[t]{2}{*}{ GGgCD78 } & I & 294 & U4-A-66 & II nt (3') & & & U87 & ATGI6LI (intron 9) \\
\hline & & & U5-U-40 & $12 \mathrm{nt}\left(5^{\prime}\right)$ & & & U87/U88 & \\
\hline GGgCD79 & 1 & 284 & U5-U-40 & I I nt (5') & & & U87/U88 & ATGI6LI (intron II) \\
\hline GGgCD80 & I & 342 & U5-C-45 & II nt (3') & & & U85/U89 & NCAPD2 (intron 3) \\
\hline GGgCD8I & I & 120 & U6-A-47 & II nt (3') & Z30 & & $\mathrm{Z30/mgU6-47}$ & mRNA CR389942 (intron I) ${ }^{3}$ \\
\hline GGgCD82 & I & 129 & U6-C-60 & $13 \mathrm{nt}\left(3^{\prime}\right)$ & & & HBII-I 66 & CKAP5 (intron 35) \\
\hline GGgCD83 & I & 163 & U6-C-62 & $10 \mathrm{nt}\left(5^{\prime}\right)$ & & & U94 & PTCD3 (intron I2) \\
\hline \multicolumn{9}{|c|}{ Orphan box C/D snoRNAs } \\
\hline GGoCDI & I & 92 & & & & & SNORDI 20 & chrl_34.43 (intron I) ${ }^{3}$ \\
\hline GGoCD2 & I & 104 & & & & & SNORDI 25 & APIBI (intron I4) \\
\hline GGoCD3 & I & 109 & & & & & HBII-295 & $\mathrm{RC} 3 \mathrm{H} 2$ (intron 5) \\
\hline GGoCD4 & I & 108 & & & & & & mRNA BX932828 (intron 2) \\
\hline GGoCD5 & 1 & 97 & & & & & & $\mathrm{DKCl}$ (intron 5) \\
\hline GGoCD6 & I & 97 & & & & & & $\mathrm{DKCl}$ (intron 9) \\
\hline GGoCD7 & I & 93 & & & & & U73b & RPS3A (intron 5) \\
\hline GGoCD8 & 2 & 87 & & & & & SNORDI 23 & chr2_I7.I (intron 5)3 \\
\hline GGoCD9 & I & 107 & & & & & & MED24 (intron I4) \\
\hline GGoCDIO & I & 93 & & & & & UIOI & RPSI 2 (intron 3) \\
\hline
\end{tabular}

'Only one isoform (isoform a) and the conserved guiding function (s) are given when the box C/D snoRNA has more than one variant; ${ }^{2}$ All host genes of chicken snoRNA paralogs are given; ${ }^{3}$ The snoRNA is transcribed in the opposite direction of its predicted host gene; Antisense element: the length of the antisense element is indicated in nucleotides, followed by its location in the box $D^{\prime}\left(5^{\prime}\right)$ and box $D\left(3^{\prime}\right)$ motifs of the snoRNA; Iso: the numbers of isoforms; Len: length of the snoRNA gene (as the CDseeker program extends 5 ' and 3' stems by $15 \mathrm{nt}$, the predicted snoRNAs may be $20 \mathrm{nt}$ larger than the snoRNAs in vivo); IR: intergenic region. 
Table 2: Box H/ACA snoRNAs in chicken

\begin{tabular}{|c|c|c|c|c|c|c|c|c|}
\hline \multirow{2}{*}{$\begin{array}{l}\text { SnoRNA } \\
\text { ID' }\end{array}$} & \multirow[t]{2}{*}{ Iso } & \multirow{2}{*}{$\begin{array}{l}\text { Len } \\
\text { (nt) }\end{array}$} & \multirow[t]{2}{*}{ Modification } & \multirow[t]{2}{*}{ Antisense element } & \multicolumn{3}{|c|}{ Functional homologs } & \multirow[t]{2}{*}{ Host gene/annotation ${ }^{2}$} \\
\hline & & & & & Yeast & Plant & Mammal & \\
\hline \multirow[t]{2}{*}{ GGgACAI } & 2 & 133 & $5.8 \mathrm{~S}-55$ & $7+5 \mathrm{nt}\left(5^{\prime}\right)$ & & & U72 & $\begin{array}{l}\text { RPL30 (intron 3); } \\
\text { EST CK607839 (intron I) }\end{array}$ \\
\hline & & & $28 S-3342$ & $7+5 \mathrm{nt}\left(5^{\prime}\right)$ & & & E2/ACA8 & \\
\hline GGgACA2 & I & $|3|$ & $5.8 S-69$ & $8+4$ nt $\left(5^{\prime}\right)$ & Pus7p & & U69 & RPL39 (intron 2) \\
\hline \multirow[t]{2}{*}{ GGgACA3 } & i & 135 & $18 \mathrm{~S}-34$ & $6+6 \mathrm{nt}\left(5^{\prime}\right)$ & snR44 & & ACA50/ACA62 & CNOTI (intron I6) \\
\hline & & & $18 S-105$ & $6+3 \mathrm{nt}\left(3^{\prime}\right)$ & snR44 & & $\begin{array}{l}\text { ACA } 36 / \mathrm{B} / \mathrm{ACA} 50 / \\
\text { ACA } 62\end{array}$ & \\
\hline GGgACA4 & 3 & $|3|$ & $18 S-36$ & $7+4 \mathrm{nt}\left(5^{\prime}\right)$ & & & U69/ACA55 & PABPC4 (intron 6,7,10) \\
\hline \multirow[t]{3}{*}{ GGgACA5 } & 1 & $|3|$ & $18 S-36$ & $4+5 \mathrm{nt}\left(3^{\prime}\right)$ & & & U69/ACA55 & $\begin{array}{l}\text { ENSGALESTG000000332 } \\
05 \text { (intron 7) }\end{array}$ \\
\hline & & & 18S-783 & $6+3 \mathrm{nt}\left(3^{\prime}\right)$ & $\operatorname{snR}|6|$ & & ACA44 & \\
\hline & & & $28 \mathrm{~S}-3926$ & $5+6 \mathrm{nt}\left(3^{\prime}\right)$ & & & ACAI & \\
\hline \multirow[t]{3}{*}{ GGgACA6 } & 1 & 135 & $18 S-120$ & $4+6 \mathrm{nt}\left(5^{\prime}\right)$ & $\operatorname{snR} 49$ & & U66 & UBAP2L (intron 14) \\
\hline & & & $28 S-1612$ & $3+7 \mathrm{nt}\left(5^{\prime}\right)$ & snR8 & & ACA56 & \\
\hline & & & $28 \mathrm{~S}-3333$ & $5+6 \mathrm{nt}\left(5^{\prime}\right)$ & snR9 & & ACA58/B & \\
\hline \multirow[t]{2}{*}{ GGgACA7 } & 1 & 128 & $185-209$ & $7+5$ nt $\left(5^{\prime}\right)$ & & & ACA28/ACA3I & $\begin{array}{l}\text { ENSGALESTG000000327 } \\
55 \text { (intron 5) }\end{array}$ \\
\hline & & & $28 S-3223$ & $6+7 \mathrm{nt}\left(3^{\prime}\right)$ & & & ACA3I & \\
\hline GGgACA8 & I & 134 & $18 \mathrm{~S}-367$ & $8+3 \mathrm{nt}\left(3^{\prime}\right)$ & & snoR86 & $\mathrm{U} 7 \mathrm{la} / \mathrm{b} / \mathrm{c} / \mathrm{d}$ & PRSI7 (intron 3) \\
\hline GGgACA9 & I & 135 & $18 S-610$ & $5+8 \mathrm{nt}\left(5^{\prime}\right)$ & & & ACA46 & CNOTI (intron 24) \\
\hline \multirow[t]{2}{*}{ GGgACAIO } & I & $|3|$ & $18 S-612$ & $3+7$ nt (5') & & & ACA20 & TCPI (intron 9) \\
\hline & & & U2-91 & $8+4 \mathrm{nt}\left(5^{\prime}\right)$ & & & & \\
\hline GGgACAII & 1 & 132 & $|8 S-76|$ & $7+5 \mathrm{nt}\left(5^{\prime}\right)$ & & & ACA25 & $\begin{array}{l}\text { ENSGALESTG000000332 } \\
05 \text { (intron I 2) }\end{array}$ \\
\hline GGgACAI2 & I & $|3|$ & I8S-774 & $6+5 \mathrm{nt}\left(5^{\prime}\right)$ & & & ACA25/ACA63 & RANBPI (intron 5) \\
\hline \multirow[t]{2}{*}{ GGgACA I3 } & I & 132 & $18 S-782$ & $7+5 \mathrm{nt}\left(5^{\prime}\right)$ & $\operatorname{snR}|6|$ & & ACA44 & RPL2I (intron 4) \\
\hline & & & $28 S-3204$ & $8+6 \mathrm{nt}\left(5^{\prime}\right)$ & & & ACA27 & \\
\hline \multirow[t]{2}{*}{ GGgACAI4 } & I & 130 & $18 S-775$ & $8+5 \mathrm{nt}\left(5^{\prime}\right)$ & snR80 & snoR9l & ACA28 & CCT6A (intron 3) \\
\hline & & & U12-19 & $7+4 \mathrm{nt}\left(3^{\prime}\right)$ & & & ACA68 & \\
\hline \multirow[t]{2}{*}{ GGgACAI5 } & 1 & 127 & $18 S-775$ & $7+4 \mathrm{nt}\left(5^{\prime}\right)$ & snR80 & snoR9l & ACA28 & EIF5 (intron 5) \\
\hline & & & I8S-824 & $7+4$ nt (3') & & & ACA28 & \\
\hline GGgACAI 6 & 1 & 130 & $|8 S-82|$ & $5+7 \mathrm{nt}\left(5^{\prime}\right)$ & & & ACA24 & $\begin{array}{l}\text { mRNA CR389003 } \\
\text { (intron I) }\end{array}$ \\
\hline \multirow[t]{4}{*}{ GGgACAI7 } & I & $|3|$ & $|8 S-82|$ & $7+7 \mathrm{nt}\left(5^{\prime}\right)$ & & & ACA24 & EIF3SIO (intron 9) \\
\hline & & & $28 S-1710$ & $6+3 \mathrm{nt}\left(5^{\prime}\right)$ & & & ACA7B & \\
\hline & & & $28 S-3128$ & $6+5 \mathrm{nt}\left(3^{\prime}\right)$ & & snoR87 & ACAI 9 & \\
\hline & & & $28 S-3219$ & $6+6 \mathrm{nt}\left(5^{\prime}\right)$ & & & ACA 19 & \\
\hline \multirow[t]{2}{*}{ GGgACA 8} & I & 122 & $185-927$ & $3+6 \mathrm{nt}\left(5^{\prime}\right)$ & & & $\mathrm{ACA} / 4 \mathrm{~b} / \mathrm{a}$ & CNOTI (intron 3I) \\
\hline & & & U2-58 & $3+8 \mathrm{nt}\left(3^{\prime}\right)$ & & & & \\
\hline GGgACA 19 & 1 & 128 & $18 S-1132$ & $10+3 \mathrm{nt}\left(5^{\prime}\right)$ & & & ACA40 & UBAP2 (intron 27) \\
\hline GGgACA20 & I & 127 & $185-1132$ & $7+4 \mathrm{nt}\left(3^{\prime}\right)$ & & & ACA 40 & $\begin{array}{l}\text { ENSGALESTG000000332 } \\
05 \text { (intron 6) }\end{array}$ \\
\hline \multirow[t]{2}{*}{ GGgACA2I } & I & 136 & $185-1196$ & $8+6 \mathrm{nt}\left(3^{\prime}\right)$ & snR85 & & $\mathrm{ACA} 5 / 5 \mathrm{~b} / \mathrm{c}$ & AP2BI (intron I) $)^{3}$ \\
\hline & & & $18 S-1197$ & $7+7 \mathrm{nt}\left(3^{\prime}\right)$ & & & $\mathrm{ACA} 5 / 5 \mathrm{~b} / \mathrm{c}$ & \\
\hline GGgACA22 & 2 & $14 \mid$ & $185-1196$ & $8+3 \mathrm{nt}\left(5^{\prime}\right)$ & snR85 & & $\mathrm{ACA} 5 / 5 \mathrm{~b} / \mathrm{c}$ & TBRG4 (intron 2,6) \\
\hline & & & $18 S-1197$ & $7+4 \mathrm{nt}\left(5^{\prime}\right)$ & & & $\mathrm{ACA} 5 / 5 \mathrm{~b} / \mathrm{c}$ & \\
\hline & & & $18 S-1580$ & $7+4 \mathrm{nt}\left(3^{\prime}\right)$ & & & $\mathrm{ACA} 5 / 5 \mathrm{~b} / \mathrm{c}$ & \\
\hline GGgACA23 & I & 135 & $185-1196$ & $7+3 \mathrm{nt}\left(5^{\prime}\right)$ & snR85 & & $\mathrm{ACA} 5 / 5 \mathrm{~b} / \mathrm{c}$ & TBRG4 (intron 7) \\
\hline GGgACA24 & 2 & 132 & $18 S-1202$ & $9+3 \mathrm{nt}\left(5^{\prime}\right)$ & snR36 & & ACA36/B & $\begin{array}{l}\text { DKCI (intron 6); } \\
\text { RFWD2 (intron 13) }\end{array}$ \\
\hline GGgACA25 & I & 133 & $18 S-1206$ & $5+6 \mathrm{nt}\left(5^{\prime}\right)$ & snR35 & & $\mathrm{ACA} 13$ & EST BU236609 (intron 2) \\
\hline GGgACA26 & I & 128 & $18 S-1305$ & $7+5 \mathrm{nt}\left(3^{\prime}\right)$ & snR83/RUF3 & & ACA4 & EIF4A2 (intron 9) \\
\hline GGgACA27 & I & 132 & $18 S-1400$ & $8+6 \mathrm{nt}\left(5^{\prime}\right)$ & & & U67 & $\mathrm{DKCl}$ (intron I0) \\
\hline & & & $28 S-1612$ & $9+4 \mathrm{nt}\left(5^{\prime}\right)$ & snR8 & & ACA56 & \\
\hline GGgACA28 & I & 133 & $18 S-1598$ & $7+5 \mathrm{nt}\left(5^{\prime}\right)$ & & & ACA4I & EEFIB2 (intron 4) \\
\hline GGgACA29 & I & 147 & $28 S-1612$ & $6+3 \mathrm{nt}\left(5^{\prime}\right)$ & snR8 & & ACA56 & CHD4 (intron 33) \\
\hline & & & U5-43 & $4+6 \mathrm{nt}\left(3^{\prime}\right)$ & & & ACA57 & \\
\hline GGgACA30 & I & 130 & $28 S-1612$ & $6+4 \mathrm{nt}\left(3^{\prime}\right)$ & snR8 & & ACA56 & EIF4A2 (intron 8) \\
\hline
\end{tabular}


Table 2: Box H/ACA snoRNAs in chicken (Continued)

\begin{tabular}{|c|c|c|c|c|c|c|c|c|}
\hline & & & $28 \mathrm{~S}-3875$ & $4+5 \mathrm{nt}\left(3^{\prime}\right)$ & & & E3 & \\
\hline & & & $28 S-3976$ & $4+7 \mathrm{nt}\left(5^{\prime}\right)$ & snR37 & & SNORA82/ACAIO & \\
\hline \multirow[t]{2}{*}{ GGgACA3I } & 1 & 130 & $28 S-1618$ & $5+5 \mathrm{nt}\left(5^{\prime}\right)$ & snR43 & & ACA9 & $\begin{array}{l}\text { mRNA CR39|354 } \\
\text { (intron 2) }\end{array}$ \\
\hline & & & $28 S-170 \mid$ & $6+4 \mathrm{nt}\left(3^{\prime}\right)$ & snR33 & & ACA9 & \\
\hline GGgACA32 & 1 & 132 & $28 S-1664$ & $6+7 \mathrm{nt}\left(5^{\prime}\right)$ & snR5 & snoR8I & ACA52 & RPLP2 (intron 2) \\
\hline GGgACA33 & 1 & 138 & $28 S-1699$ & $6+4 \mathrm{nt}\left(5^{\prime}\right)$ & & & $\mathrm{HBI}-\mathrm{II} 5$ & $\begin{array}{l}\text { mRNA CR523I } 68 \\
\text { (intron I) }\end{array}$ \\
\hline GGgACA34 & 1 & 123 & $28 S-178 \mid$ & $7+4 \mathrm{nt}\left(5^{\prime}\right)$ & snR5 & & ACA32 & $\begin{array}{l}\text { ENSGALESTG000000332 } \\
05 \text { (intron II) }\end{array}$ \\
\hline GGgACA35 & I & 134 & $28 S-2269$ & $8+3 \mathrm{nt}\left(5^{\prime}\right)$ & & & ACA6I & EST CK9868I0 (intron 3) \\
\hline \multirow[t]{2}{*}{ GGgACA36 } & I & 156 & $28 S-3126$ & $5+6 \mathrm{nt}\left(5^{\prime}\right)$ & snR3 & & ACA6 & RPSA (intron 3) \\
\hline & & & $28 S-3886$ & $5+5 \mathrm{nt}\left(5^{\prime}\right)$ & & & ACA2I & \\
\hline \multirow[t]{2}{*}{ GGgACA37 } & I & 123 & $28 S-3128$ & $5+5 \mathrm{nt}\left(3^{\prime}\right)$ & & snoR87 & ACAI9 & EIF3SIO (intron 8) \\
\hline & & & U6-86 & $6+5 \mathrm{nt}\left(5^{\prime}\right)$ & & & ACA65 & \\
\hline GGgACA38 & 1 & 134 & $28 \mathrm{~S}-3223$ & $9+5 \mathrm{nt}\left(3^{\prime}\right)$ & & & АСАЗІ & $\begin{array}{l}\text { ENSGALESTG000000327 } \\
55 \text { (intron 6) }\end{array}$ \\
\hline GGgACA39 & 2 & 127 & $28 S-3311$ & $4+7 \mathrm{nt}\left(5^{\prime}\right)$ & & & ACA54 & NAPIL4 (intron 7,9) \\
\hline GGgACA40 & I & 153 & $28 S-3340$ & $6+5 \mathrm{nt}\left(5^{\prime}\right)$ & & & E2 & RPSA (intron 4) \\
\hline GGgACA4I & 1 & $|3|$ & $28 S-3409$ & $7+3 \mathrm{nt}\left(5^{\prime}\right)$ & & & $\mathrm{ACA} 3$ & RPL27A (intron 3) \\
\hline GGgACA42 & I & 130 & $28 S-3448$ & $5+4 \mathrm{nt}\left(3^{\prime}\right)$ & & & $\mathrm{ACA} 3$ & RPL27A (intron 2) \\
\hline \multirow[t]{2}{*}{ GGgACA43 } & 1 & 132 & $28 S-375 I$ & $8+4 \mathrm{nt}\left(5^{\prime}\right)$ & & & & $\mathrm{IR}$ \\
\hline & & & $28 S-3816$ & $3+6 \mathrm{nt}\left(3^{\prime}\right)$ & & & ACA23 & \\
\hline GGgACA44 & 2 & 145 & $28 S-3754$ & $6+3 \mathrm{nt}\left(3^{\prime}\right)$ & & & ACA34 & KIAA20I3 (intron I); IR \\
\hline \multirow[t]{2}{*}{ GGgACA45 } & I & $13 \mid$ & $28 S-3886$ & $6+5 \mathrm{nt}\left(5^{\prime}\right)$ & & & ACA2I & RPL23 (intron 3) \\
\hline & & & $28 S-3955$ & $6+4 \mathrm{nt}\left(3^{\prime}\right)$ & snRIO & snoR74 & ACA2I & \\
\hline \multirow[t]{2}{*}{ GGgACA46 } & I & 135 & $28 S-3858$ & $7+3 \mathrm{nt}\left(5^{\prime}\right)$ & & & U65 & RPLI2 (intron 4) \\
\hline & & & $28 S-3912$ & $6+5 \mathrm{nt}\left(3^{\prime}\right)$ & snR34 & U65 & U65 & \\
\hline GGgACA47 & 1 & 136 & $28 S-4393$ & $5+4 \mathrm{nt}\left(5^{\prime}\right)$ & & & U64/ACA22 & RPS2 (intron 5) \\
\hline GGgACA48 & 1 & 139 & U2-34 & $5+4 \mathrm{nt}\left(5^{\prime}\right)$ & & & U92 & Cl4orfI59 (intron I) \\
\hline \multirow[t]{2}{*}{ GGgACA49 } & I & 129 & U2-39 & $7+4 \mathrm{nt}\left(3^{\prime}\right)$ & & & ACA26 & $\begin{array}{l}\text { mRNA CR52382I } \\
\text { (intron I) }\end{array}$ \\
\hline & & & U2-4I & $6+7 \mathrm{nt}\left(5^{\prime}\right)$ & & & ACA26 & \\
\hline \multirow[t]{2}{*}{ GGgACA50 } & I & 129 & U2-43 & $7+7 \mathrm{nt}\left(3^{\prime}\right)$ & & & U92 & RCJMB04_9g9 (intron 12) \\
\hline & & & U2-44 & $7+8 \mathrm{nt}\left(3^{\prime}\right)$ & & & U92 & \\
\hline \multirow[t]{2}{*}{ GGgACA5I } & I & 129 & U2-54 & $6+3 \mathrm{nt}\left(3^{\prime}\right)$ & & & U93 & EST BUI 27305 (intron 2) \\
\hline & & & U5-53 & $6+4 \mathrm{nt}\left(5^{\prime}\right)$ & & & U93 & \\
\hline GGgACA52 & I & 147 & U6-86 & $6+4 \mathrm{nt}\left(3^{\prime}\right)$ & & & ACA65 & HTR2C (intron I) ${ }^{3}$ \\
\hline \multicolumn{9}{|c|}{ Orphan box H/ACA snoRNAs } \\
\hline GGoACAI & I & 136 & & & & & & RPLI8A (intron 2) \\
\hline GGoACA2 & I & 130 & & & & & ACAI 8 & $\begin{array}{l}\text { ENSGALESTG000000332 } \\
05 \text { (intron 9) }\end{array}$ \\
\hline GGoACA3 & 1 & 138 & & & & & ACA8 & $\begin{array}{l}\text { ENSGALESTG000000332 } \\
05 \text { (intron I0) }\end{array}$ \\
\hline GGoACA4 & I & 137 & & & & & UI00 & TIPIN (intron 6) \\
\hline GGoACA5 & 1 & 126 & & & & & & KIAA0907 (intron 6) \\
\hline GGoACA6 & 1 & $13 \mid$ & & & & & ACA29 & TCPI (intron I) \\
\hline GGoACA7 & I & 129 & & & & & & GARNLI (intron 4) \\
\hline GGoACA8 & I & 140 & & & & & & CWFI9LI (intron I2) \\
\hline GGoACA9 & I & $13 \mid$ & & & & & & NOL5A (intron 3) \\
\hline GGoACAIO & I & 132 & & & & & & WHSCI (intron 17) \\
\hline
\end{tabular}

'Only one isoform (isoform a) and the conserved guiding function (s) are given when the box H/ACA snoRNA has more than one variant; ${ }^{2}$ All host genes of chicken snoRNA paralogs are given; ${ }^{3}$ The snoRNA is transcribed in the opposite direction of its predicted host gene; Antisense element: the length of the antisense element is indicated in nucleotides, followed by its location in the ' $\Psi$ pocket' of the first hairpin ( 5 ') and second hairpin

(3') of the snoRNA; Iso: the numbers of isoforms; Len: length of the snoRNA gene predicted; IR: intergenic region.

within our expected sizes were detected under stringent conditions of PCR. Notably, the expression of four snoRNAs (GGgCD20, GGgCD76, GGgACA10 and GGgACA43) that are predicted to guide modification sites that have been previously reported to lack potential guide
snoRNAs was detected. Two snoRNAs (GGgCD11a and GGgACA46) hosted within the ribosomal protein gene family exhibit a robust signal; whereas the other 12 snoRNAs, most of which are not located within house keeping genes, were detected only weakly. It remains to be further 
A

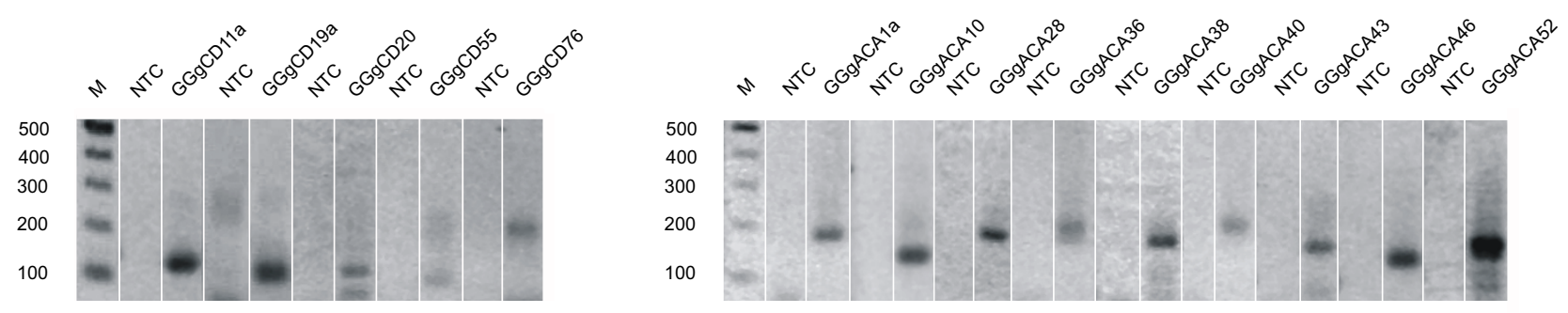

B
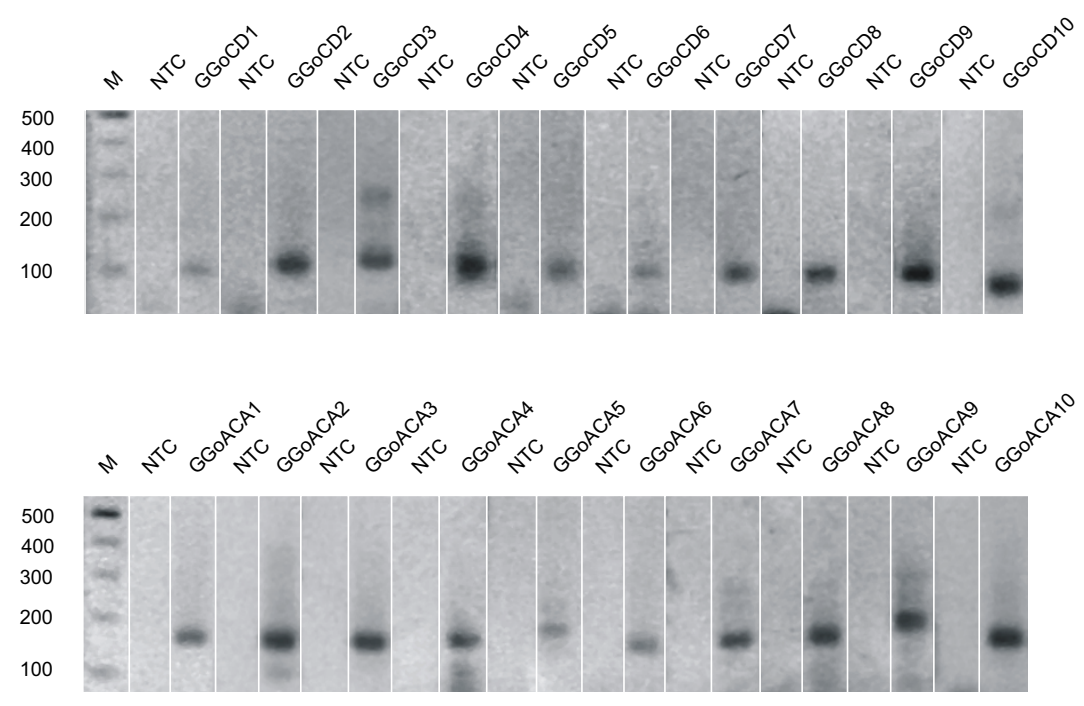

Figure 2

RT-PCR analysis of snoRNAs amplified from small RNA cDNAs of chicken embryos at stage HH34. A DNA ladder on each side indicates the size of the fragments. NTC, Non-template control. M, DNA marker. (A) Expression of the five guide box C/D snoRNAs and nine guide boxH/ACA snoRNAs. (B) Expression of the ten orphan box C/D snoRNAs and ten orphan box H/ACA snoRNAs.

tested whether the different intensities of the electrophoresis bands observed under the same PCR conditions reflect the different expression levels of the snoRNAs in vivo. We next applied this method to detect the 20 orphan snoRNAs predicted in this study. All these orphan snoRNAs were amplified and the sizes of the PCR products were consistent with their predicted sizes (Figure 2B). Our experimental analysis by RT-PCR demonstrated that the snoRNA genes predicted in this study are expressed.

\section{Clustered snoRNAs most often show conserved synteny between humans and chickens}

Almost all of the snoRNAs identified in the chicken are located in introns of known genes or spliced expressed sequence tags (ESTs), which is consistent with previous reports in mammals $[1,9,23]$. The majority of chicken snoRNAs (80\%) appear on chromosomes 1, 2, 4, 5, 8, 10 and $Z$. None of the snoRNA genes was located on chromosomes $21,22,26,29,30,31$ and $\mathrm{W}$.
From this study, a total of 98 box C/D and 38 box H/ACA snoRNAs are organized into 44 clusters (see Additional file 5). Eleven clusters carry both box C/D and box H/ACA snoRNAs, and the other clusters harbor exclusively either box $\mathrm{C} / \mathrm{D}$ or box H/ACA snoRNAs. Five clusters reside in the introns of non-protein-coding genes or ESTs or novel transcripts, and the remaining 39 clusters are located in protein-coding HGs. Genomic analysis was carried out on these clustered snoRNAs and their HGs. Eighty-six of 136 clustered snoRNAs $(63 \%)$ in chicken are syntenic to human, and 36 protein-coding HGs are the orthologs of the human HGs. Four types of snoRNA clusters were determined depending on the synteny characteristics of the clustered snoRNAs between chicken and human (Figure 3).

The Type-1 snoRNA clusters (clusters 1-14) show perfect synteny between chicken and human (Figure 3A). The HGs of these clustered snoRNAs in chicken are ortholo- 
A. Type-1 snoRNA cluster

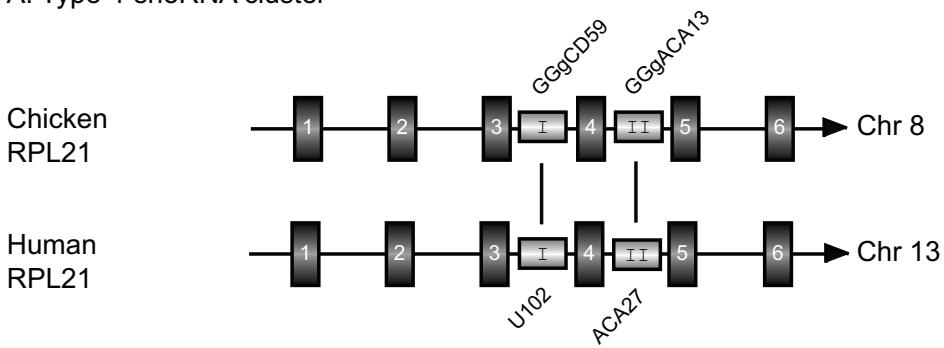

B. Type-2 snoRNA cluster

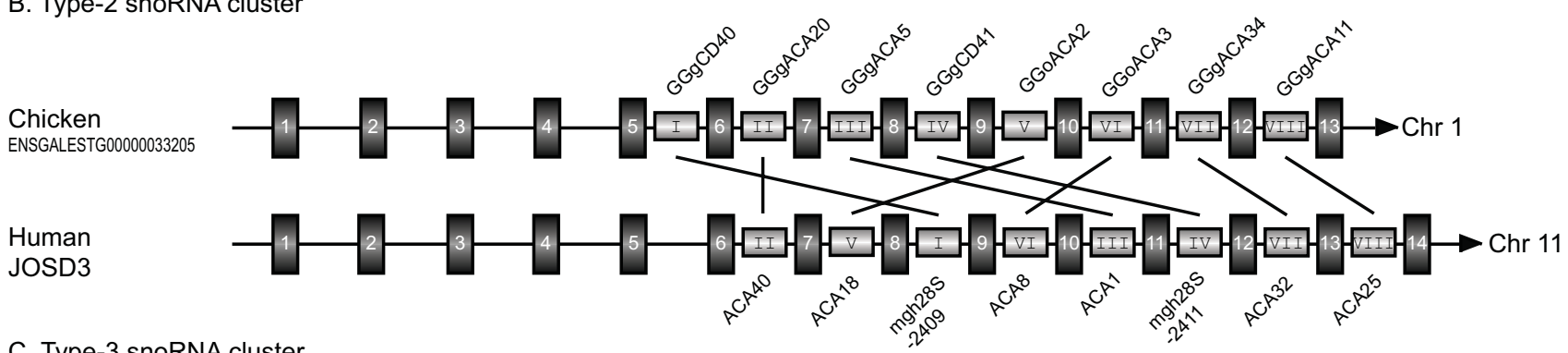

C. Type-3 snoRNA cluster

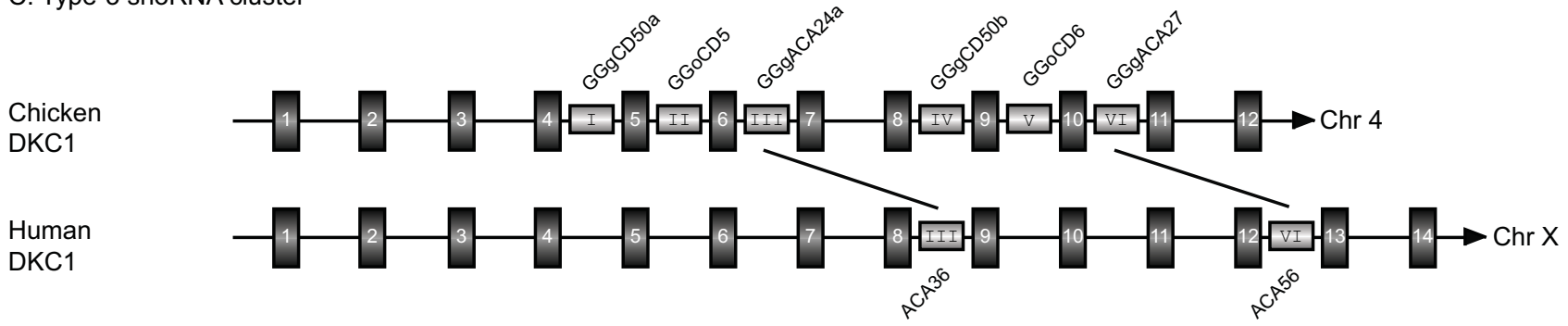

D. Type-4 snoRNA cluster

Chicken

KPNB1

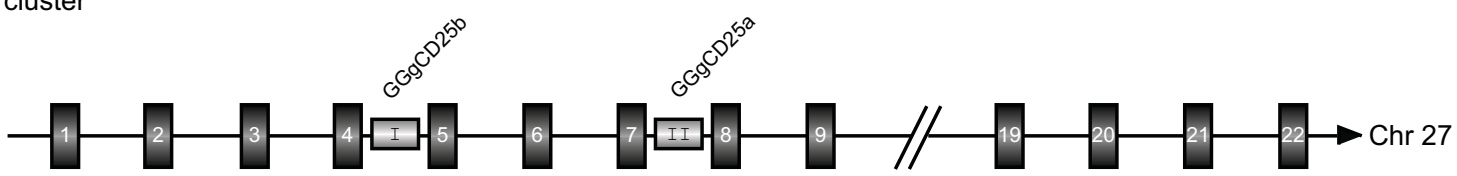

Figure 3

Comparative analyses of the four types of snoRNA clusters between chicken and human. Only one example is presented in each type of snoRNA cluster (A-D). The exons and snoRNA regions (not drawn to scale) are denoted by black and gray boxes, respectively. The snoRNA names, HGs and their chromosomal locations are indicated. The cognate snoRNAs between chicken and human are indicated by the same Roman numerals and linked by a line. The exon regions are indicated by Arabic numerals in order. The transcriptional direction is indicated by the arrowhead.

gous to those in human, indicating that they are derived from common ancestral genes over 310 million years ago.

The Type-2 snoRNA clusters (clusters 15-33) are conserved in content but neither in snoRNA copy numbers nor in order. Fifteen HGs of the type-2 snoRNA clusters are orthologous between chicken and human, and the other snoRNA clusters reside in either non-protein-coding genes, novel transcripts or the ESTs of which their mRNA sequences show low or no similarity between the two species. Interestingly, the HG of cluster 15 is an Ensemble novel protein coding gene (ENSGALESTG00000033205) and tandemly encodes multiple chicken counterparts of human snoRNAs (ACA40, ACA18, mgh28S-2409, ACA8,
ACA1, mgh28S-2411, ACA32 and ACA25) located within different introns of the same host gene JOSD3 (Figure 3B). However, these eight clustered chicken snoRNAs are arrayed in a different order when compared to those of the human and other vertebrates, which might have resulted from lineage-specific intragenic translocation (see Additional file 6). Sequence comparison revealed that the mRNA sequence of ENSGALESTG00000033205 showed low similarity (45\%) to that of JOSD3, which is in sharp contrast to the high similarity of the snoRNA counterpart between the two species.

The Type-3 snoRNA clusters (clusters 34-37) have HGs that are orthologous to human genes but with the inser- 
tion of new snoRNAs. For example, four novel snoRNAs are inserted in cluster 34 when compared to that in human, and the HGs (DKC1) between chicken and human are orthologous (Figure 3C).

The Type-4 snoRNA clusters (clusters 38-44) have independently evolved in the chicken (Figure 3D). Corresponding snoRNAs could not be identified in human orthologous regions, suggesting that these snoRNA clusters might have changed their HGs during chicken and human divergence.

\section{A gas5-like non-protein-coding snoRNA transcript found in the chicken}

Whilst characterizing the synteny of the clustered snoRNAs between the chicken and human, we noted that snoRNA cluster 26 is of special interest, being located in an HG (mRNA CR387333) whose genomic organization is similar to that of human and mouse gas 5 (growth arrestspecific transcript 5) [24], a non-protein-coding snoRNA HG. Both contain short exons $(<100 \mathrm{nt})$ and tandem array snoRNA counterparts in the corresponding intronic regions (Figure 4A). With the exception of the chicken counterpart of human U77 (in intron 4) that is replaced by two cognate snoRNAs of human U80 (GGgCD36a and GGgCD36b in introns 4 and 5 respectively), the other snoRNA counterparts array in the same order on their corresponding HGs of the chicken and human. The gene encoding mRNA CR387333 has a typical TATA box (TATATAA) and a 5'TOP sequence (TCTgCCTTTCCgCCCCT) at position -14 to +3 , indicating that it is also a member of the 5' TOP gene family [25]. Comparison of the two transcripts (mRNA CR387333 and gas5) revealed that their sequence similarity varied significantly with

\section{A}

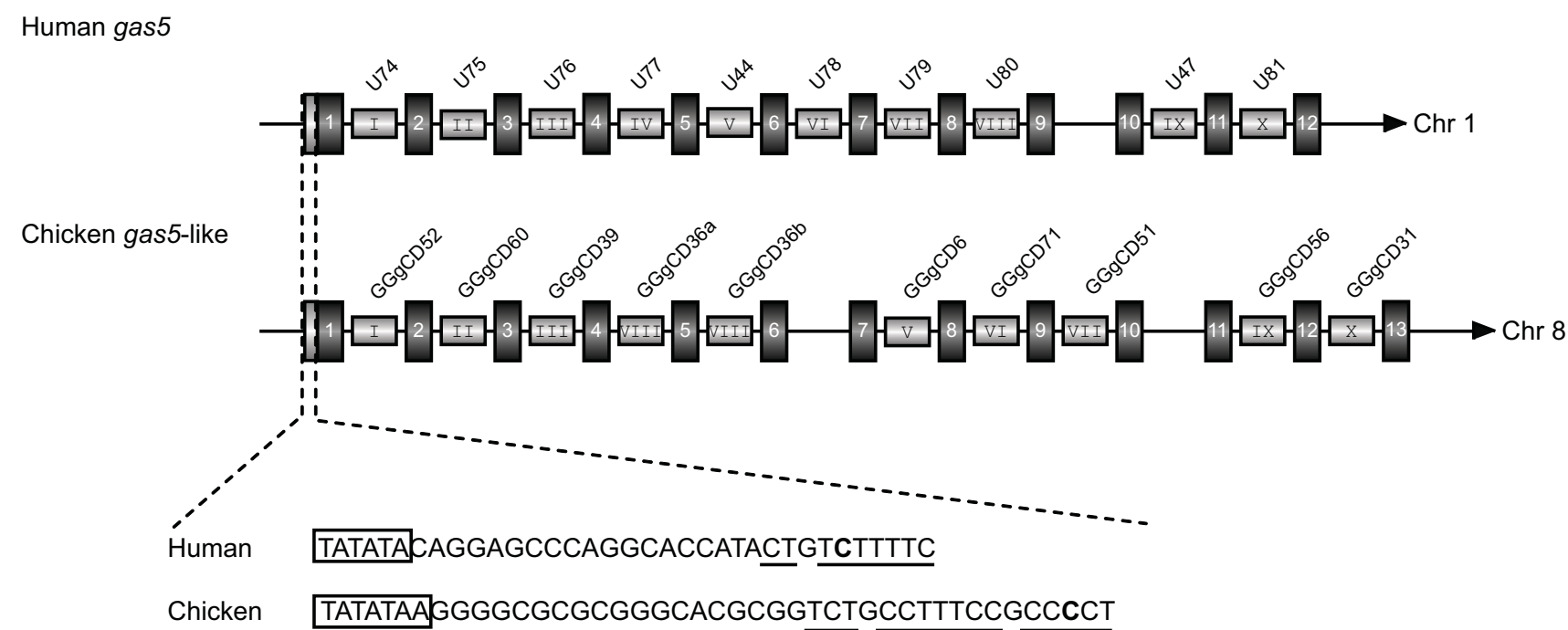

B
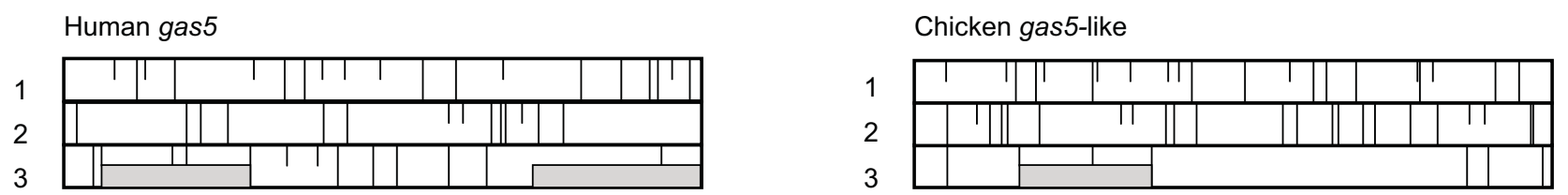

Figure 4

Comparison of the structure of snoRNAs encoded in the human gas5 and chicken gas5-like host gene (A) and analysis of the protein-coding potential of human (Accession No. AF I 4I346) and chicken (mRNA CR387333) transcripts (B). (A) The exons and snoRNA regions (not drawn to scale) are denoted by black and gray boxes, respectively. The snoRNA names are indicated. The snoRNA counterparts between chicken and human are indicated by the same Roman numerals. The exon regions are indicated by Arabic numerals in order. The TATA elements are boxed; the transcription start sites are indicated in bold and the 5' TOP sequences are underlined. (B) Coding potential of human and chicken transcripts identified by the BLAST ORF Finder http://www.ncbi.nlm.nih.gov/projects/gorf/gorf.html. Short and long vertical bars represent start and stop codons, respectively, in all three frames. The gray boxes denote the most likely reading frames. 
each pair of corresponding portions. The most highly conserved regions are the snoRNA sequences, whereas the regions not encoding snoRNA are much more discrepant $(42 \%)$ between chicken and human. Furthermore, the presence of only short ORFs and numerous stop codons suggests the low probability of protein coding (Figure 4B), which is similar to gas5. Therefore, the gene encoding mRNA CR387333 is classified as a gas5-like non-proteincoding snoRNA HG, and the intron-encoded snoRNAs may be the only functional portions of the transcript.

\section{Structural and functional evolution of chicken snoRNAs}

Comparative functional analyses have revealed that many guide snoRNAs involved in rRNA posttranscriptional modification are phylogenetically conserved in mammals. However, extensive recombination and separation of guiding function are also discerned in the chicken snoRNAs when compared with the human snoRNAs. For example, human box C/D snoRNAs U36A and U36C possess the conserved function as guides for the 2'-O-ribose methylation of $18 \mathrm{~S}$ rRNA-A668 and 28S rRNA-A3703 [26], respectively, whereas both of the corresponding sites in the chicken rRNAs are predicted to be guided by the single snoRNA GGgCD18a, as well as its paralog GGgCD18b (Figure 5A). A similar case of recombination can be found in the box H/ACA snoRNA GGgACA29 which is predicted to guide the $\Psi$ s at $28 \mathrm{~S}$ rRNA- $\Psi 1612$ and snRNA U5- 443 , while the two corresponding modifications are reported to be independently guided by human snoRNAs ACA56 and ACA57, respectively $[16,27,28]$. In contrast, the human snoRNA U32A/B and U69 are double guiders and potentially guide two methylations and two $\Psi$ s respectively, whereas the four corresponding modification functions are separately possessed by four single guiders in the chicken (Figure 5B).

It is worth noting that the cooperative evolution of the sequences and guiding function sites can be found in some of the chicken snoRNAs. A case in point is the GGgACA30 and its human cognate E3 snoRNA. Although high sequence similarity ( $75 \%)$ was found between the two counterparts, GGgACA30 potentially guides the $28 \mathrm{~S}$ rRNA- $\Psi 1612,-\Psi 3875$ and $-\Psi 3976$, whereas E3 is predicted to simply guide the $28 \mathrm{~S}$ rRNA- $\Psi 4390$ (correspond-
A

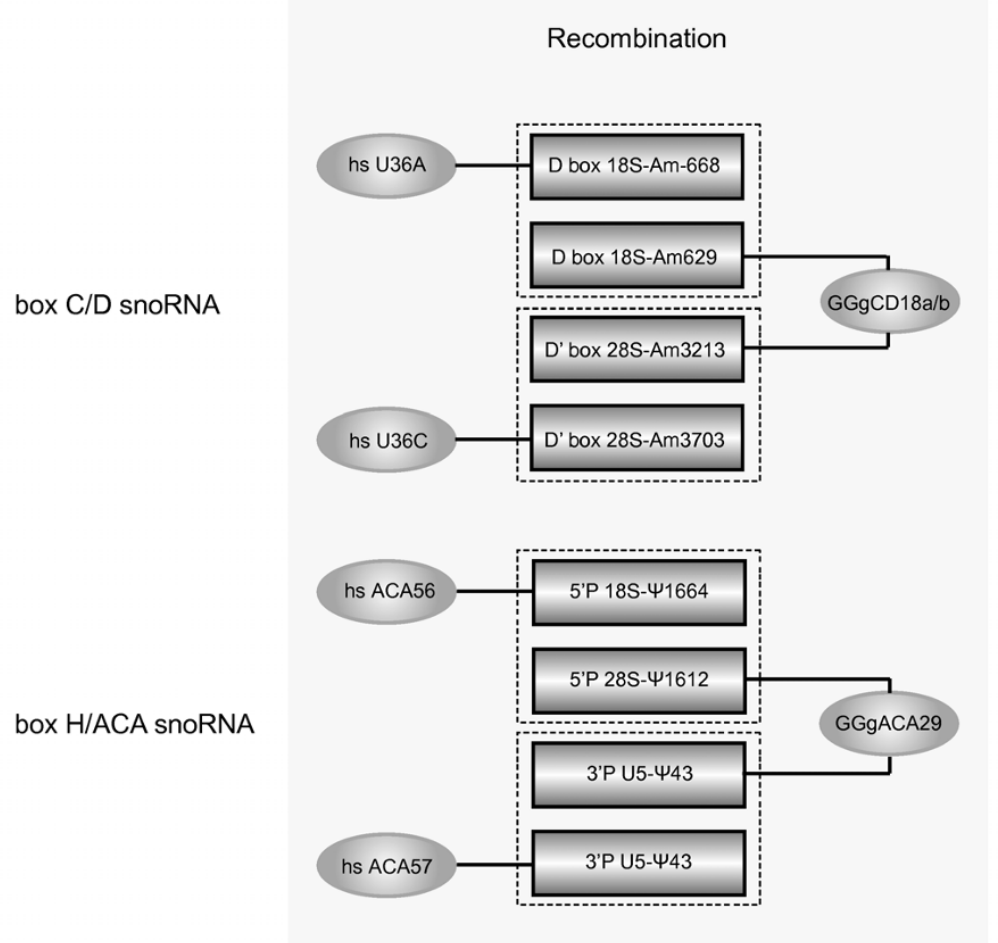

B

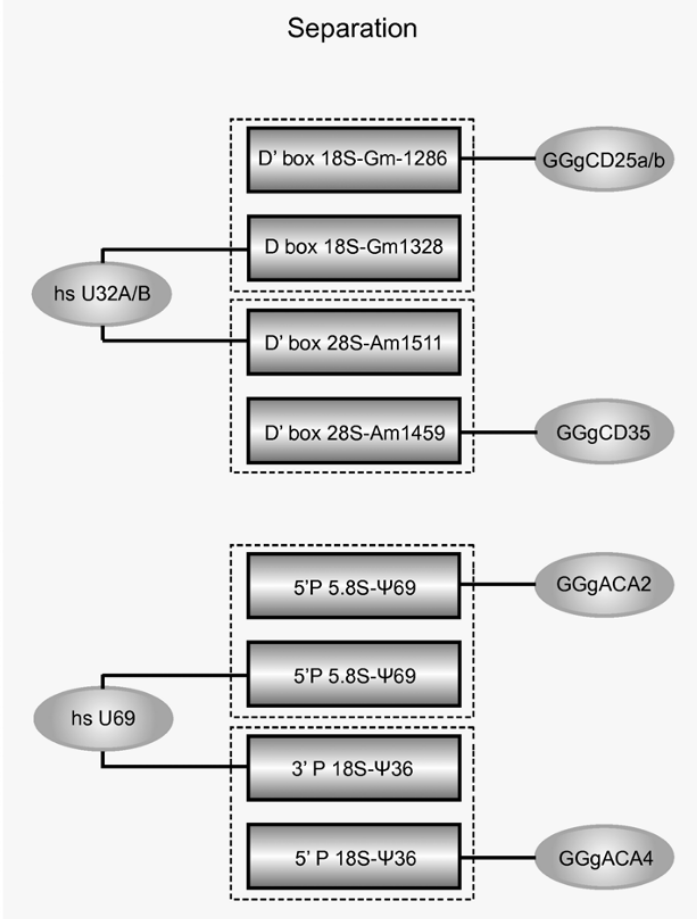

\section{Figure 5}

Schematic representation of the structural and functional evolution discerned in the chicken and human snoRNAs. (A) Recombination of guiding function. (B) The separation of guiding function. The names of snoRNAs and their guiding functions are indicated in the oval and closed boxes, respectively, and are linked by line. The cognate guiding elements in the chicken and human snoRNAs are boxed by a dashed line. 
ing to chicken 28S rRNA- 43875 ) [29]. The analysis of functional loci has revealed that loss of the other two guiding functions for $\mathrm{E} 3$ results from extensive nucleotide substitutions and indels, disrupting the snoRNA/substrate RNA base-pairing potential of the $5^{\prime}$ and 3 ' regions in the snoRNA. In contrast, the chicken cognate presents a perfect $\Psi$-guiding domain and functional motif. Another example is GGgACA14 as a guider for the $\Psi$ s of the U1219 and $18 \mathrm{~S}$ rRNA-775, whereas its human counterpart ACA22 is predicted to guide the $\Psi$ s at different residues of rRNA (28S rRNA- 44966 [16] and - $\Psi 4975$ [17]). In addition, nucleotide variation can also destroy the guiding function and results in the conversion of guide snoRNAs to orphan snoRNAs, such as GGoACA3 versus the human cognate ACA8 snoRNA.

\section{Discussion A model for the prediction of functional snoRNAs in the genome}

Comparative analysis of genomes between closely or distantly related species might provide limited information on conserved regions [30]. The chicken bridges the evolutionary gap between mammals and other vertebrates and represents an intermediate-level comparison for the human, making it very useful for detecting functional elements with high specificity [19]. As a family of the most abundant and important noncoding RNAs, many snoRNAs were found to be conserved in different organisms. In this study, we have provided a detailed catalog of chicken snoRNAs to understand snoRNA gene repertoire differences between avian and other vertebrate lineages. A total of 201 gene variants encoding 93 box C/D and 62 box H/ ACA snoRNAs were identified in the chicken genome. In contrast to extensive functional and/or pseudogene paralogs found in mammals $[17,31]$, the majority of snoRNAs in chicken remain singletons, whereas some other novel paralogs are produced by duplication and seem specific to the chicken lineage. Notably, clustered snoRNAs show a large degree of conserved synteny between chicken and human, which greatly simplifies classification of the chicken orthologs of human snoRNAs. Unlike the eutherian mammals with tandem repeated snoRNAs in the imprinted regions [32,33], none of the imprinted snoRNA orthologs are found in the chicken. Our result might provide some clues to support that imprinting seems to have evolved in therians and hence has only been confirmed in marsupials and eutherian mammals [34-36]. Intriguingly, most of the imprinted snoRNAs in mammals are orphan snoRNAs and constitute new members of the 'dark matter' in the RNA world. With the exception of snoRNA HBII-52 that is reported to be involved in RNA editing [37] and alternative splicing [38], the function of the other orphan snoRNAs in mammals remains enigmatic. In our study, we also identified 20 orphan snoRNAs that are expressed in chicken embryos and conserved in different vertebrate species. As with microRNAs, the conservation of snoRNAs among species suggests that they bear conserved biological functions. These orphan snoRNAs might therefore be subject to purifying selection and hence are predicted to be functional in still unknown biological processes.

\section{Intragenic duplication and divergence dominated in the expansion of novel snoRNAs in the chicken}

Diverse molecular mechanisms are involved in the creation of new gene (protein-coding gene) structures, such as gene duplication and retroposition [39]. Compared with protein-coding genes, little is known about the creation of novel npcRNA genes in the genome. To our knowledge, two main strategies are responsible for the generation of most of the novel snoRNA paralogs in vertebrates. The first strategy is that some snoRNA paralogs are generated by intragenic duplication where the snoRNAs are tandemly duplicated within the same gene, a process termed cis-duplication $[12,13]$. In many cases, the sequence and the secondary structure of these snoRNA paralogs are highly conserved. The second strategy is where snoRNAs may duplicate and insert into a new host gene or a paralogous host gene in a different genomic location, and is termed trans-duplication [12,13]. In this study, we observed that almost all of the chicken snoRNA paralogs are generated via intragenic duplication, and similar cases are also found in other vertebrates including platypus snoRNAs [13]. There is only one case of five GGgCD61 paralogs created by the combination of intragenic and intergenic duplication during the chicken snoRNA expansion. Most of the novel snoRNA paralogs ( $18 \%)$ emerge in the adjacent intron regions where the corresponding loci in other mammals lack the cognate snoRNAs, which is in contrast to the few snoRNAs ( $~ 7 \%)$ found in platypus [13]. The sequences of many snoRNA paralogs have undergone extensive nucleotide variation, but the guiding function regions and the conserved structures are maintained, indicating that strong purifying selection is acting upon them. However, the nucleotide substitutions and insertions or deletions (indels) might also endow the snoRNAs with novel guiding functions (such as GGgACA30 and GGgACA14) or destroy the guiding function (such as GGoACA3), depending on whether or not a perfect functional domain is present within the sequence variation. Recently, hundreds of snoRNAs derived from non-autonomous retroposition have been reported in the human $[10,11]$ and platypus genomes [31], revealing a new dimension in the evolution of novel snoRNAs. However, there is no evident trace of the snoRNA-retroposonlike counterparts found in the chicken genome (data not shown), which is consistent with the paucity of functional genes formed by retroposition [19]. Therefore, based on both our results and information obtained from other vertebrate genome analyses, snoRNAs derived from retroposition may originate from mammals. Intragenic duplication and divergence might be the major driving 
force responsible for expansion of novel snoRNAs in the chicken genome.

\section{A hotspot of snoRNA gene expansion in the gas5-like non- protein-coding snoRNA HG}

Non-protein-coding HGs encoding snoRNAs are unusual because they do not appear to specify protein products and snoRNAs may be the only functional portions of the transcripts [40]. Different members of the non-proteincoding HGs encoding snoRNAs (UHG, gas5, U17HG, U19HG, U50HG) have been reported in humans and mice [24,40-43], and even in fruit flies [44]. Non-protein-coding HGs have not been found in Caenorhabditis elegans, which suggests a different strategy adopted in higher metazoa for regulating snoRNA expression [45]. Among all the members of non-protein-coding HGs, gas5 is of interest because of its large snoRNA-coding capability and deviant accumulation in cells undergoing serum starvation or density arrest [24]. In this study, we detect the gas5like HG which also lacks protein-coding potential but instead encodes 10 box C/D snoRNAs within its introns. Unexpectedly, we could not find any other non-proteincoding HGs and corresponding snoRNAs in the chicken genome. Comparative analyses of the snoRNA content in the gas5 and other gas5-like HGs reveal diverse snoRNA numbers and gene orders in different vertebrates. In this study, nine human cognate snoRNAs were identified in the chicken gas5-like HG. However, the human U77 counterpart within the fourth intron is replaced by its cognate U80 snoRNA (GGgCD36a), and the other U80 paralog (GGgCD36b) is tandemly arrayed in the next intron, which leads to a different gene order compared to human (Figure 4A). Our comparative analyses of the snoRNAs in gas5 of vertebrates indicted that the mammalian U77 snoRNA might evolve from the other non-mammalian vertebrate cognate U80 snoRNA which has undergone mutation and lost the function of guiding adenine methylation at the corresponding site of $28 \mathrm{~S}$ rRNA. In addition, a novel snoRNA-like sequence is also detected in intron 6 and found to be chicken-specific, suggesting the process of snoRNA expansion is actively ongoing. Intriguingly, similar cases of extensive snoRNA gene tandem duplication and intragenic transposition can be detected in the corresponding HGs of other vertebrates, such as Danio rerio and Xenopus tropicalis (data not shown). Although the mechanism by which snoRNA sequences become embedded in the introns of their HGs is still enigmatic, the gas5-like HGs appears to be a hotspot for gaining snoRNA novelties in vertebrates.

\section{Conclusion}

This is the first genome-wide and systematic screen for snoRNAs in the chicken by applying a computational package and experimental methods. The characteristics of the guiding function and genomic organization of the chicken snoRNAs were extensively compared with that of the human counterparts. We have provided a detailed catalog of chicken snoRNAs to understand snoRNA gene repertoire differences between avian and other vertebrate lineages. Our results improve annotation of the 'darkness matter' in the npcRNA world of the vertebrate genome and provide a unique perspective into snoRNA evolution in vertebrates.

\section{Methods \\ Data sources}

Ten chicken-vertebrate pairwise alignments (galGal3/ hg18, galGal3/mm8, galGal3/rn4, galGal3/equCab1, galGal3/monDom4, galGal3/ornAna1, galGal3/anoCar1, galGal3/xenTro2, galGal3/danRer4 and galGal3/fr2) whole-genome alignment (WGA) sequences and the Zebra finch (Taeniopygia guttata) sequence data (taeGut1) were downloaded from the UCSC Genome Bioinformatics site http://genome.ucsc.edu. The repeat families were removed by RepeatMasker http://www.repeatmasker.org/ PreMaskedGenomes.html. Sequences and annotation data for known human snoRNA genes (which were used in program training) were downloaded from snoRNALBME-db on March 2008 as references for chicken snoRNAs http://www-snorna.biotoul.fr/[4]. UCSC KnownGene, RefGene, Genscan and Ensembl annotation for chicken protein genes and transcript units were downloaded from the UCSC Genome Bioinformatics site and Ensembl Genome Browser http://www.ensembl.org/.

The chicken 28S and 18S rRNA gene sequences (see Additional files 7) were obtained by combining experimental and bioinformatical approaches. The 5.8S rRNA and snRNA (U1-U6, and U12) sequences were retrieved from the UCSC Genome Bioinformatics Site based on similarity search. The 2'-O-methylation and pseudouridylation sites of human rRNAs and snRNAs were mapped to the chicken rRNA and snRNA sequences.

\section{snoRNA searching strategies and sequence analyses}

We systematically searched the chicken genome for snoRNAs with 10 whole-genome alignments (WGA) using snoSeeker http://genelab.zsu.edu.cn/snoseeker/ as described previously [7]. For box C/D snoRNAs, the $15 \mathrm{nt}$ flanking sequences of known snoRNA were extracted for folding the terminal stem and training (the CDseeker program extends 5' and 3' stems of the snoRNA sequences by $15 \mathrm{nt}$ ). For box H/ACA snoRNAs, the maximum tail length was $3 \mathrm{nt}$ downstream of the ACA box. The potential targets (2'-O-methylation and $\Psi$ ) in rRNAs and snRNAs were also determined using snoSeeker. For the comparative analyses of vertebrate snoRNAs, we used the BLAT and the convert track of the UCSC Genome Brower. The human snoRNA-HGs are used as the reference for retrieving the chicken counterparts using the UCSC convert track. Next, the introns of chicken HGs were extracted as a dataset for searching the snoRNA candidates using sno- 
Seeker. The synteny analysis of snoRNA genes in the chicken and human genomes was also implemented on the UCSC Genome Brower.

RNA isolation and construction of snoRNA CDNA libraries Total cellular RNA was isolated from stage HH34 chicken embryos by the guanidine thiocyanate/phenol-chloroform procedure described by Chomoczynski et al. [46]. The construction of two families of snoRNA cDNA libraries was performed as described previously [18] with little revision. Briefly, $10 \mu \mathrm{g}$ total RNA was polyadenylated using a poly(A) polymerase (Takara) at $30^{\circ} \mathrm{C}$ for $20 \mathrm{~min}$ and subsequently reverse-transcribed into the firststranded cDNAs using $\left[\gamma^{32} \mathrm{P}\right] \mathrm{dATP}$ labeled anchor primer $\mathrm{dT}_{16}$-TGT (for box H/ACA snoRNA) and $\mathrm{dT}_{16}$-TCAG (for box CD snoRNA) and MMLV reverse transcriptase (Promega) at $42^{\circ} \mathrm{C}$ for $1 \mathrm{hr}$. The reaction mixture was sizefractioned on a denaturing $10 \%$ polyacrylamide gel ( $8 \mathrm{M}$ urea and $1 \times$ TBE buffer). cDNAs with sizes ranging from 90 to $180 \mathrm{nt}$ (for box CD snoRNA) and from 140 to 170 nt (for box H/ACA snoRNA) were excised and eluted from the gel in $0.3 \mathrm{M} \mathrm{NaCl}$ buffer. The selected cDNA was tailed with dGTP at the 3 ' end by using terminal deoxynucleotidyl transferase (Takara) at $37^{\circ} \mathrm{C}$ for $30 \mathrm{~min}$. G-tailed cDNAs were then amplified by PCR with a forward primer $\mathrm{dT}_{23} \mathrm{H}_{2}$ and a reverse primer polyCM. A snoRNA-specific primer and a universal reverse primer polyCM were used for amplification of each of the snoRNAs. The routine PCR protocol $\left(94^{\circ} \mathrm{C}\right.$ for $5 \mathrm{~min}$, then 30 cycles of $94^{\circ} \mathrm{C}$ for $1 \mathrm{~min}, 50^{\circ} \mathrm{C}$ for $1 \mathrm{~min}, 72^{\circ} \mathrm{C}$ for $1 \mathrm{~min}$, and $72^{\circ} \mathrm{C}$ for 5 min) was used. A $5 \mu \mathrm{l}$ sample of each PCR product was analyzed on a $2.5 \%$ agarose gel.

\section{Oligodeoxynucleotides}

Oligonucleotides for PCR-based detection and primers used for PCR of the chicken $18 \mathrm{~S}$ and $28 \mathrm{~S}$ rRNA genes were synthesized by Invitrogen Co. (Shanghai, China) and are shown in Additional file 8 . The primers used in the reverse transcription reaction were 5 ' end-labeled with $\left[\gamma-{ }^{32} \mathrm{P}\right]$ ATP (Yahui Co.) and subjected to purification according to standard laboratory protocols.

\section{Abbreviations}

snoRNAs: Small nucleolar RNAs; npcRNAs: non-proteincoding RNAs; scaRNA: small Cajal body-specific RNA; snRNA: spliceosomal nuclear RNA; $\Psi$ : pseudouridylation; RT-PCR: reversed transcript PCR; HG: host gene; EST: expressed sequence tag; indel: insertion or deletion; ORF: open reading frame; WGA: whole-genome alignment; cDNA: complementary DNA.

\section{Authors' contributions}

PS and LHQ conceived the study and contributed to manuscript writing. JHY and PS collected the data and carried out the data analyses. PS performed the experiments. HZ and DGG assisted in experimental design and the data analysis, respectively. All authors read and approved the final manuscript.

\section{Additional material}

\section{Additional file 1}

Functional prediction of the chicken snoRNAs. The data provided represent the functional prediction of the chicken box $C / D(A)$ and box $\mathrm{H} /$ ACA snoRNAs (B).

Click here for file

[http://www.biomedcentral.com/content/supplementary/14712164-10-86-S1.pdf]

\section{Additional file 2}

Sequences of snoRNA genes predicted in Gallus gallus. The data show the sequences of snoRNA genes predicted in Gallus gallus. Structural elements of snoRNAs are boxed.

Click here for file

[http://www.biomedcentral.com/content/supplementary/1471-

2164-10-86-S2.pdf]

\section{Additional file 3}

Comparative analysis of chicken snoRNAs and their counterparts in six other vertebrates. The data provided represent the comparative analysis of chicken snoRNAs and their counterparts in human, mouse, opossum, platypus, lizard and frog.

Click here for file

[http://www.biomedcentral.com/content/supplementary/1471-

2164-10-86-S3.xls]

\section{Additional file 4}

Strategy for construction of specialized cDNA libraries enriched in box $C / D(A)$ and box H/ACA snoRNAs (B). The figure shows the strategy for constrction of specialized cDNA libraries enriched in box $C / D(A)$ and box H/ACA snoRNAs (B).

Click here for file

[http://www.biomedcentral.com/content/supplementary/14712164-10-86-S4.pdf]

\section{Additional file 5}

Synteny analysis of the 44 snoRNA clusters between chicken and human. The data provided represent the synteny analysis of the 44 snoRNA clusters between chicken and human.

Click here for file

[http://www.biomedcentral.com/content/supplementary/14712164-10-86-S5.xls]

\section{Additional file 6}

Schematic illustration of lineage-specific intragenic translocation of the snoRNA cluster 15. The figure shows the schematic illustration of lineage-specific intragenic translocation of the snoRNA cluster 15.

Click here for file

[http://www.biomedcentral.com/content/supplementary/14712164-10-86-S6.pdf]

\section{Additional file 7}

Sequences of the chicken $28 S$ and $18 S$ rRNA genes. The data show the sequences of the chicken $28 S$ rRNA and $18 S$ rRNA genes obtained by combining computational and experimental methods.

Click here for file

[http://www.biomedcentral.com/content/supplementary/14712164-10-86-S7.pdf] 


\section{Additional file 8}

Sequences of oligonucleotides for RT-PCR based experiment and primers for PCR of chicken $18 S$ and $28 S$ rRNA genes. The data show all the sequences of oligonucleotides for RT-PCR based experiment and primers for PCR of chicken $18 S$ and $28 S$ rRNA genes.

Click here for file

[http://www.biomedcentral.com/content/supplementary/14712164-10-86-S8.xls]

\section{Acknowledgements}

This research was supported by the National Basic Research Program (No. 2005CB724600), the funds from the Ministry of Education of China and Guangdong Province (No. IRT0447, NSF05200303) and the National Natural Science Foundation of China (30570398, 3077I I5I, 30830066). We thank three anonymous referees who made valuable suggestions that led to considerable improvements in the paper.

\section{References}

I. Kiss T: Small nucleolar RNAs: an abundant group of noncoding RNAs with diverse cellular functions. Cell 2002, 109(2): $145-148$

2. Matera AG, Terns RM, Terns MP: Non-coding RNAs: lessons from the small nuclear and small nucleolar RNAs. Nat Rev Mol Cell Biol 2007, 8(3):209-220.

3. Darzacq X, Jady BE, Verheggen C, Kiss AM, Bertrand E, Kiss T: Cajal body-specific small nuclear RNAs: a novel class of 2'-O-methylation and pseudouridylation guide RNAs. Embo J 2002, 2I (I I):2746-2756.

4. Lestrade L, Weber MJ: snoRNA-LBME-db, a comprehensive database of human H/ACA and C/D box snoRNAs. Nucleic Acids Res 2006:DI58-162.

5. Bachellerie JP, Cavaille J, Huttenhofer A: The expanding snoRNA world. Biochimie 2002, 84(8):775-790.

6. Cavaille J, Buiting K, Kiefmann M, Lalande M, Brannan $\mathrm{Cl}$, Horsthemke $B$, Bachellerie JP, Brosius J, Huttenhofer A: Identification of brainspecific and imprinted small nucleolar RNA genes exhibiting an unusual genomic organization. Proc Natl Acad Sci USA 2000, 97(26): 143||$-|43| 6$.

7. Yang JH, Zhang XC, Huang ZP, Zhou H, Huang MB, Zhang S, Chen YQ, Qu LH: snoSeeker: an advanced computational package for screening of guide and orphan snoRNA genes in the human genome. Nucleic Acids Res 2006, 34( I 8):5 I I 2-5 I 23.

8. Brown JW, Echeverria M, Qu LH: Plant snoRNAs: functional evolution and new modes of gene expression. Trends Plant Sci 2003 $8(1): 42-49$.

9. Smith CM, Steitz JA: Sno storm in the nucleolus: new roles for myriad small RNPs. Cell I997, 89(5):669-672.

10. Weber MJ: Mammalian small nucleolar RNAs are mobile genetic elements. PLoS Genet 2006, 2(I 2):e205

II. Luo Y, Li S: Genome-wide analyses of retrogenes derived from the human box H/ACA snoRNAs. Nucleic Acids Res 2007, 35(2):559-57I.

12. Zemann A, op de Bekke A, Kiefmann M, Brosius J, Schmitz J: Evolution of small nucleolar RNAs in nematodes. Nucleic Acids Res 2006, 34(9):2676-2685.

13. Schmitz J, Zemann A, Churakov G, Kuhl H, Grutzner F, Reinhardt R, Brosius J: Retroposed SNOfall-a mammalian-wide comparison of platypus snoRNAs. Genome Res 2008, 18(6): 1005-1010.

14. Griffiths-Jones S: Annotating noncoding RNA genes. Annu Rev Genomics Hum Genet 2007, 8:279-298.

15. Huttenhofer A, Kiefmann M, Meier-Ewert S, O'Brien J, Lehrach $\mathrm{H}$ Bachellerie JP, Brosius J: RNomics: an experimental approach that identifies $20 \mathrm{I}$ candidates for novel, small, non-messenger RNAs in mouse. Embo J 200I, 20(I I):2943-2953.

16. Kiss AM, Jady BE, Bertrand E, Kiss T: Human box H/ACA pseudouridylation guide RNA machinery. Mol Cell Biol 2004, 24(13):5797-5807.
17. Schattner P, Barberan-Soler S, Lowe TM: A computational screen for mammalian pseudouridylation guide H/ACA RNAs. Rna 2006, I 2(I): I5-25.

18. Gu AD, Zhou H, Yu CH, Qu LH: A novel experimental approach for systematic identification of box H/ACA snoRNAs from eukaryotes. Nucleic Acids Res 2005, 33(22): e 194.

19. Consortium ICGS: Sequence and comparative analysis of the chicken genome provide unique perspectives on vertebrate evolution. Nature 2004, 432(70 I 8):695-716

20. Kiss AM, Jady BE, Darzacq X, Verheggen C, Bertrand E, Kiss T: A Cajal body-specific pseudouridylation guide RNA is composed of two box H/ACA snoRNA-like domains. Nucleic Acids Res 2002, 30(2I):4643-4649.

21. Ro S, Park C, Jin J, Sanders KM, Yan W: A PCR-based method for detection and quantification of small RNAs. Biochem Biophys Res Commun 2006, 35 I (3):756-763.

22. Ro S, Park C, Sanders KM, McCarrey JR, Yan W: Cloning and expression profiling of testis-expressed microRNAs. Dev Biol 2007, 3 I I (2):592-602.

23. Brown JW, Marshall DF, Echeverria M: Intronic noncoding RNAs and splicing. Trends Plant Sci 2008, I3(7):335-342.

24. Smith CM, Steitz JA: Classification of gas 5 as a multi-smallnucleolar-RNA (snoRNA) host gene and a member of the 5'terminal oligopyrimidine gene family reveals common features of snoRNA host genes. Mol Cell Biol 1998, I 8( I 2):6897-6909.

25. Amaldi F, Pierandrei-Amaldi P: TOP genes: a translationally controlled class of genes including those coding for ribosomal proteins. Prog Mol Subcell Biol 1997, I 8: I- 17.

26. Nicoloso M, Qu LH, Michot B, Bachellerie JP: Intron-encoded, antisense small nucleolar RNAs: the characterization of nine novel species points to their direct role as guides for the 2'O-ribose methylation of rRNAs. I Mol Biol 1996, 260(2): 178-195.

27. Ofengand J, Bakin A: Mapping to nucleotide resolution of pseudouridine residues in large subunit ribosomal RNAs from representative eukaryotes, prokaryotes, archaebacteria, mitochondria and chloroplasts. J Mol Biol 1997, 266(2):246-268.

28. Krol A, Gallinaro H, Lazar E, Jacob M, Branlant C: The nuclear 5S RNAs from chicken, rat and man. U5 RNAs are encoded by multiple genes. Nucleic Acids Res 198I, 9(4):769-787.

29. Ganot $P$, Bortolin ML, Kiss T: Site-specific pseudouridine formation in preribosomal RNA is guided by small nucleolar RNAs. Cell 1997, 89(5):799-809.

30. Furlong RF: Insights into vertebrate evolution from the chicken genome sequence. Genome Biol 2005, 6(2):207.

31. Schmitz J, Zemann A, Churakov G, Kuhl H, Grutzner F, Reinhardt R, Brosius ]: Retroposed SNOfall - a mammalian-wide comparison of platypus snoRNAs. Genome Res 2008, 18(6): I005-1010.

32. Seitz H, Royo H, Lin SP, Youngson N, Ferguson-Smith AC, Cavaille J: Imprinted small RNA genes. Biol Chem 2004, 385( I 0):905-9II.

33. Cavaille J, Seitz H, Paulsen M, Ferguson-Smith AC, Bachellerie JP: Identification of tandemly-repeated C/D snoRNA genes at the imprinted human $14 q 32$ domain reminiscent of those at the Prader-Willi/Angelman syndrome region. Hum Mol Genet 2002, I I(I3): I527-I538.

34. Wilkins JF, Haig D: What good is genomic imprinting: the function of parent-specific gene expression. Nat Rev Genet 2003 , 4(5):359-368.

35. Edwards CA, Rens W, Clarke O, Mungall AJ, Hore T, Graves JA, Dunham I, Ferguson-Smith AC, Ferguson-Smith MA: The evolution of imprinting: chromosomal mapping of orthologues of mammalian imprinted domains in monotreme and marsupial mammals. BMC Evol Biol 2007, 7:157.

36. Warren WC, Hillier LW, Marshall Graves JA, Birney E, Ponting CP, Grutzner F, Belov K, Miller W, Clarke L, Chinwalla AT, et al: Genome analysis of the platypus reveals unique signatures of evolution. Nature 2008, 453(7192): 175- I83.

37. Vitali P, Basyuk E, Le Meur E, Bertrand E, Muscatelli F, Cavaille J, Huttenhofer A: ADAR2-mediated editing of RNA substrates in the nucleolus is inhibited by C/D small nucleolar RNAs. J Cell Biol 2005, I 69(5):745-753.

38. Kishore $S$, Stamm S: The snoRNA HBII-52 regulates alternative splicing of the serotonin receptor 2C. Science 2006, 3II (5758):230-232. 
39. Long M, Betran E, Thornton K, Wang W: The origin of new genes: glimpses from the young and old. Nat Rev Genet 2003, 4(I I):865-875.

40. Tycowski KT, Shu MD, Steitz JA: A mammalian gene with introns instead of exons generating stable RNA products. Nature 1996, 379(6564):464-466.

41. Pelczar P, Filipowicz W: The host gene for intronic UI7 small nucleolar RNAs in mammals has no protein-coding potential and is a member of the 5'-terminal oligopyrimidine gene family. Mol Cell Biol 1998, 18(8):4509-45।8.

42. Bortolin ML, Kiss T: Human UI9 intron-encoded snoRNA is processed from a long primary transcript that possesses little potential for protein coding. Rna 1998, 4(4):445-454.

43. Tanaka R, Satoh H, Moriyama M, Satoh K, Morishita Y, Yoshida S, Watanabe T, Nakamura Y, Mori S: Intronic U50 small-nucleolarRNA (snoRNA) host gene of no protein-coding potential is mapped at the chromosome breakpoint $t(3 ; 6)(q 27 ; q 15)$ of human B-cell lymphoma. Genes Cells 2000, 5(4):277-287.

44. Huang ZP, Zhou H, He HL, Chen CL, Liang D, Qu LH: Genomewide analyses of two families of snoRNA genes from Drosophila melanogaster, demonstrating the extensive utilization of introns for coding of snoRNAs. Rna 2005, II(8): $1303-13 \mid 6$

45. Huang ZP, Chen CJ, Zhou H, Li BB, Qu LH: A combined computational and experimental analysis of two families of snoRNA genes from Caenorhabditis elegans, revealing the expression and evolution pattern of snoRNAs in nematodes. Genomics 2007, 89(4):490-50I.

46. Chomczynski P, Sacchi N: The single-step method of RNA isolation by acid guanidinium thiocyanate-phenol-chloroform extraction: twenty-something years on. Nat Protoc 2006, I(2):58I-585.

Publish with Bio Med Central and every scientist can read your work free of charge

"BioMed Central will be the most significant development for disseminating the results of biomedical research in our lifetime. "

Sir Paul Nurse, Cancer Research UK

Your research papers will be:

- available free of charge to the entire biomedical community

- peer reviewed and published immediately upon acceptance

- cited in PubMed and archived on PubMed Central

- yours - you keep the copyright

Submit your manuscript here:

http://www.biomedcentral.com/info/publishing_adv.asp
BioMedcentral 